\title{
Flow dynamics between two concentric counter-rotating porous cylinders with radial through-flow
}

\author{
Sebastian Altmeyer ${ }^{*} *$ \\ Castelldefels School of Telecom and Aerospace Engineering, Universitat Politècnica de Catalunya, \\ 08034 Barcelona, Spain
}

\begin{abstract}
(Received 26 July 2019; accepted 25 October 2021; published 20 December 2021)
This paper investigates the impact of radial mass flux on Taylor-Couette flow in counterrotating configuration, in which a Hopf bifurcation gives rise to branches of nontrivial solutions. Using direct numerical simulation we elucidate structures, dynamics, stability, and bifurcation behavior in qualitative and quantitative detail as a function of inner Reynolds numbers $\left(\operatorname{Re}_{i}\right)$ and radial mass flux $(\alpha)$ spanning a parameter space with a rich variety of solutions. Both radial inflow and strong radial outflow stabilize the system, whereas weak radial outflow has a strong destabilizing effect. We detected the existence of stable ribbons and mixed ribbons with low azimuthal wave number without symmetry restriction. In addition, ribbon solutions and mixed-ribbon solutions can be stable or unstable saddles. Furthermore, in the case of unstable saddles alternations between two different symmetrically related saddles generate different heteroclinic cycles. For alternating stationary (in comoving frame) ribbons the persistence time in one saddle decreases with distance from the onset. The persistence time for the heteroclinic cycle of alternating mixed ribbons shows a more complicated dependence with variation in control parameters and seems to follow an intermittency scenario of type III. Depending on whether the symmetrically related solutions are stationary or time-dependent, the heteroclinic connection can be either of oscillatory or nonoscillatory type.
\end{abstract}

DOI: 10.1103/PhysRevFluids.6.124802

\section{INTRODUCTION}

For more than a century, the Taylor-Couette flow [1], flow confined by two independently driven concentric cylinders, has played a paradigmatic role for studying the effects of viscosity, for testing applications of low-dimensional dynamical systems theory, and for the development of hydrodynamic stability theory [1-4]. Its geometrical simplicity allows for well-controlled experiments as well as verification of numerical simulations and thus, to shed light on complex flow dynamics, pattern formation and finally the transition to hydrodynamic turbulence [5-9].

The present paper examines the effect of radial mass flux (injection and suction through the cylinder walls) and the resulting interaction and modifications of different stability mechanisms. This setup is related to and motivated by the use of centrifugal instabilities and the results may provide new insight for applications, such as rotating filtration [10], vortex flow reactors [11,12] flow separation devices, such as food separation or oil-sand separation in the petroleum industry [13]. Typically, these applications are based on a radial flow from the inner toward the outer cylinder of a Taylor-Couette cell [12]. To be efficient, these separating devices are usually work continuously to perform the separation, while the mixture enters at one side and the 'clean' flow exits at the other. Thus, an intrinsic axial flow is present at all times, which favors helical flow structures [1,14], which is a key motivation for the present work. The principal commercial use of

\footnotetext{
*Corresponding author: sebastian.andreas.altmeyer@upc.edu
} 
this method is the extraction of plasma from whole blood $[10,15,16]$ but it has been also proposed as a method for various industrial filtration applications [17-25]. For possible applications, aside from the knowledge of where instabilities appear in the $\left(\operatorname{Re}_{i}, \operatorname{Re}_{o}, \alpha\right)$ parameter space it is also necessary to know what the new flows look like, e.g., azimuthally dominated or helical dominated component. And an answer to this question, which itself is important, is the essential step toward our main aim. Despite the well-known differences between a radial through-flow between two porous cylinders and the generic rotating filtration in which the outer cylinder is nonporous, these studies can provide insight into the fundamental mechanisms that alter the stability of Taylor-Couette flow due to the presence of such a radial flow [26-30].

In addition, although our model is much simpler than the complex scenario for accretion disks, the study of radial mass flux on Taylor-Couette flow may have potential relevance to the fluid dynamics of astrophysical phenomena [31-33] as well to the polar vortex flow in the Earth's atmosphere [34]. In fact there are astrophysical examples in which external mass sources generate radial inflow, thus producing accretion disks. Central to these structures and dynamics are more complex typically spiraling flows, which form in situations such as motion around black holes at the centers of galaxies, in binary stars or in disks during the birth process of stars. Here the magnetorotational instability can be produced by ionization [35]. Such potential astrophysical applications beyond any specific application or device proves that the Couette flow with radial mass flux is of interest for a much wider range of problems beyond classical filtration devices.

The presence of a radial through-flow in the annulus between two differentially rotating porous cylinders modifies the absolute stability of the Taylor vortex flow [26-29,36-38]. All these works, whether experiments, linear or weakly nonlinear stability analysis, or numerical simulations, come to the same conclusion: Both converging radial flow and sufficiently strong diverging flow have a stabilizing effect on the Taylor instability, while weak to moderate diverging flow destabilizes the system $[26,38]$.

Using linear stability analysis and considering both axisymmetric and nonaxisymmetric perturbations at various radius ratios the combined effects of axial and radial flow on the stability of Taylor vortex flow has been investigated [30,38]. Martinand et al. [28] studied convective instability of radial through-flow. They observed that radial flow only slightly affects the number of helices and the critical Taylor number. For small axial flow the convective instability is axisymmetric, but convective helical modes with an increasing number of helices having a helicity opposite to that of the base flow dominate with increasing axial flow. Regarding absolute instabilities that occur at higher Reynolds numbers they detected that unstable axisymmetric modes appeared for inward radial flows, while helical absolute instability modes whose helicity is identical to that of the base flow appear for outward radial flow.

Ilin and Morgulis [39] found (in linear stability analysis) that for counter-rotating cylinders a destabilizing effect of a weak diverging flow can occur for all values of the gap between the cylinders, depending on gap width. They detected that a sufficiently strong increase in radial flow, directed either inward or outward, flattens the critical curves in the $\left(\operatorname{Re}_{i}, \operatorname{Re}_{o}\right)$ plane, which means that the stability properties of the basic flow are almost independent of $\mathrm{Re}_{o}$, i.e., the effect of the outer cylinder becomes very weak, which is in agreement with earlier results [27]. But in all these cases the primary stable bifurcating solutions are topological closed flow structures such as Taylor vortex flow (TVF) [27] and wavy Taylor vortices (wTVF) [4,14,40]. However, Gallet et al. [31] described the existence of unstable nonaxisymmetric modes in the linearized stability problem for specific parameter conditions. The recent work by Martinand et al. [29] demonstrated that for wide gap and sufficiently strong radial flow the critical mode becomes nonaxisymmetric leading to the primary appearance of helical flow structures. The same result can be achieved by sufficiently fast rotation of the outer cylinder in opposite directions. In both cases a Hopf bifurcation gives rise to branches of spiral vortex flow (spirals, SPIs) [14,41-43] and ribbons (RIB) [4,41,44-49], both appearing at a common threshold out of basic Circular Couette flow (CCF). Thus, RIB is similar to standing waves, since it is an equal superposition of two spirals moving in axially opposite 


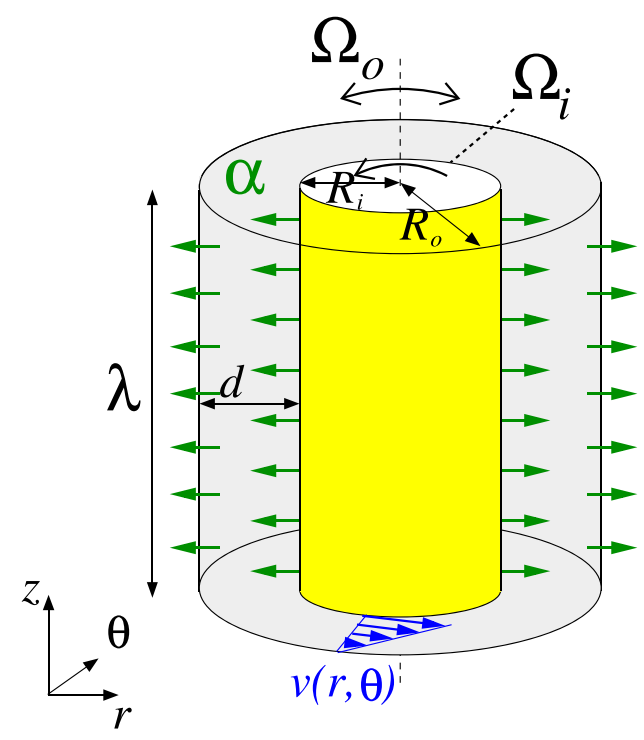

FIG. 1. Schematic of the Taylor-Couette system illustrating radial flow in counter-rotating configuration including sketch of laminar velocity profile $v(r, \theta)$ (not to scale). The radial flow can be directed outward $(\alpha>O)$ (illustrated), or inward $(\alpha<O)$.

directions, upward and downward, and as a result they are only azimuthally rotating. Topological speaking, RIB solutions are saddles in a comoving frame of reference.

Heteroclinic cycles [50,51], which are a collection of solution trajectories that connects equilibria, periodic solutions or even chaotic sets [52], are a generic feature of dynamical systems with symmetries. Such saddle-sink connections and their features have been investigated in detail in the past [53-55]. Heteroclinic cycles are robust under perturbations that preserve the system symmetries and they exist over a range of parameters.

To our knowledge a study of more complex helical structures such as SPI and their superpositions to RIB or mixed ribbons (mRIB) and their interaction under radial flow is missing. The present study provides this investigation.

The outline of the paper is as follows. Sec. II describes the basic equations and numerical method used in the present study and provides an overview of all flow structures discovered including a list of all the flows we have found. Sec. III presents numerical investigation of the bifurcation scenario via $\operatorname{Re}_{i}$ and $\alpha$ followed by an overview of all detected solutions in the $\left(\operatorname{Re}_{i}, \alpha\right)$ parameter space. Flow dynamics and spatial-temporal characteristics of various flow structures are presented focusing on alternations between unstable (m)RIB solutions and their heteroclinic connections. Finally, Sec. IV provides the discussion and conclusion.

\section{SYSTEM SETTING AND NUMERICAL PROCEDURE}

Consider the flow driven in the annular gap between two independently rotating cylinders $[1,44]$ - the Taylor-Couette system (Fig. 1). The inner cylinder of radius $R_{i}$ rotates at angular speed $\Omega_{i}$ and the outer cylinder of radius $R_{o}$ rotates at angular speed $\Omega_{o}$. In the present study, we consider in the axial direction periodic boundary conditions which are set to $\lambda /\left(R_{o}-R_{i}\right)=1.6$ ( $\lambda$ being the axial wavelength), corresponding to an axial wave number $k=(2 \pi / \lambda)=3.927$. The fluid in the annulus is assumed to be Newtonian, isothermal, and incompressible with kinematic viscosity $v$. The nondimensional Navier-Stokes equations governing the flow are

$$
\partial_{t} \mathbf{u}+(\mathbf{u} \cdot \nabla) \mathbf{u}=-\nabla p+\nabla^{2} \mathbf{u}, \quad \nabla \cdot \mathbf{u}=0,
$$


where $\mathbf{u}=(u, v, w)$ is the velocity in cylindrical coordinates $(r, \theta, z)$ and the corresponding vorticity is $\boldsymbol{\nabla} \times \mathbf{u}=(\xi, \eta, \zeta)$. The system is governed by the following independent nondimensional parameters:

Inner Reynolds number: $\quad \operatorname{Re}_{i}=\Omega_{i} R_{i} d / \nu$,

Outer Reynolds number: $\quad \operatorname{Re}_{o}=\Omega_{o} R_{o} d / \nu$,

Radial Reynolds number: $\quad \alpha=u_{i} R_{i} / \nu\left(=u_{o} R_{o} / v\right.$, owing to continuity),

Radius ratio:

$$
b=R_{i} / R_{o} \text {. }
$$

For this work we assume a wide gap with fixed radius ratio $b=0.5$. The length and time scales of the system are set by the gap width $d=R_{o}-R_{i}$ and the diffusion time $d^{2} / \nu$, respectively. The pressure in the fluid is normalized by $\rho v^{2} / d^{2}$. Additionally, the outer Reynolds numbers is fixed to $\operatorname{Re}_{o}=$ -125 , while only varying either the inner Reynolds numbers $\operatorname{Re}_{i}$ or the radial Reynolds number $\alpha$. On the cylindrical surfaces, the velocity fields are $\mathbf{u}\left(r_{i}, \theta, z, t\right)=\left(u_{i}, \operatorname{Re}_{\mathrm{i}}, 0\right)$ and $\mathbf{u}\left(r_{o}, \theta, z, t\right)=$ $\left(u_{o}, \mathrm{Re}_{\mathrm{o}}, 0\right)$ (with $\left.u_{o}=b u_{i}\right)$, respectively, where the nondimensional inner and outer radii are $r_{i}=$ $R_{i} / d$ and $r_{o}=R_{o} / d$.

The governing equations and the boundary conditions are invariant under arbitrary rotations $R_{\alpha}$ about the axis, arbitrary axial translation $Z_{l}$ and with respect to time translations $\phi_{t_{0}}$. The actions of these symmetries on the velocity field are

$$
\begin{aligned}
R_{\alpha}(u, v, w)(r, \theta, z, t) & =(u, v, w)(r, \theta+\alpha, z, t), \\
Z_{l}(u, v, w)(r, \theta, z, t) & =(u, v, w)(r, \theta, z+l, t), \\
\phi_{t_{0}}(u, v, w)(r, \theta, z, t) & =(u, v, w)\left(r, \theta, z, t+t_{0}\right) .
\end{aligned}
$$

These idealizations lead to a unique CCF basic state which depends only on $\mathrm{r}$. The system has $S O(2) \times O(2)$ symmetry, where $S O(2)$ is the group of arbitrary rotations about the axis and $O(2)$ is the group containing reflection at any height $z$ and translations in $z$. The mean, possibly zero, axial flux remains unchanged along the axial direction. At the same time all symmetries [Eq. (2.3)] are conserved with only the radial profiles of the CCF basic state depending on the parameter $\alpha$.

\section{A. Numerical method and classification}

The Navier-Stokes Eqs. (2.1) are solved using a second-order time-splitting method with consistent boundary conditions for the pressure [56,57]. Our code G1D3 [58] is a combination of a finite-difference method in the radial and axial directions $(r, z)$ and a Fourier-Galerkin expansion in the azimuthal direction $(\theta)$ with time splitting resulting in a decomposition

$$
f(r, \theta, z, t)=\sum_{m} f_{m}(r, z, t) e^{i m \theta}
$$

of all fields $f \in\{u, v, w, p\}$. For the parameter regime considered, the choice $m_{\max }=10$ (with $m$ being the azimuthal wave number) provides adequate accuracy. We use a uniform grid with spacing $\delta r=\delta z=0.02$ and time steps $\delta t<1 / 3800$. For diagnostic purposes, we also evaluate the complex mode amplitudes $f_{m, n}(r, t)$ obtained from a Fourier decomposition in the axial direction

$$
f_{m}(r, z, t)=\sum_{n} f_{m, n}(r, t) e^{i n k z}
$$

More details regarding the description of the Fourier spectrum are provided in the Appendix.

Further, as a global measure to characterize various flow structures we use the total modal kinetic energy

$$
E_{\mathrm{kin}}=\sum_{m} E_{m}=\frac{1}{2} \sum_{m} \int_{0}^{2 \pi} \int_{-\Gamma / 2}^{\Gamma / 2} \int_{r_{i}}^{r_{o}} \mathbf{u}_{m} \mathbf{u}_{m}^{*} r d r d z d \theta
$$


TABLE I. Flow state nomenclature and abbreviations. From left to right: flow M-state ${ }_{m^{*}, A \leftrightarrow B}^{D}$ (M denotes the dominant azimuthal mode), with $D$ describing the dynamics as $t$ (time-dependent), $m^{*}$ identifies the dominant modulated mode for mRIB solution and $A_{A \leftrightarrow B}$ indicates a heteroclinic cycle (hc) between two symmetrically related saddles, which are visited alternately. Furthermore the characteristics stable (s), unstable (u), alternating are identified (cf. caption of Fig. 4). The relation $\gg, \lessdot$ indicates that corresponding mode amplitudes are larger or smaller, respectively; modes indicated as $\pm m$ have identical amplitudes. Note that these modes are not necessarily the higher harmonics of the principal mode. Please see the Appendix for more details regarding the description of the Fourier spectrum.

\begin{tabular}{|c|c|c|c|}
\hline Flow & (dominant) modes $m$ & Spec./Stab. & Dynamics \\
\hline TVF & 0 & $\mathrm{~s},[\mathrm{u}]$ & - \\
\hline wTVF & $0 \pm 1$ & $\mathrm{~s}$ & rotating \\
\hline L1-[R1-]SPI & $1[-1]>-1[1]$ & $\mathrm{s},[\mathrm{u}]$ & left-[right-]winding, rotating \\
\hline 1-RIB & \pm 1 & $\mathrm{~s},[\mathrm{u}]$ & rotating \\
\hline $1-\mathrm{RIB}_{A \leftrightarrow B}$ & \pm 1 & hc & alternating $A \leftrightarrow B$, rotating \\
\hline M0-flow & 0 & $\mathrm{u}$ & transitional \\
\hline $1-\mathrm{mRIB}^{t}$ & $\pm 1 \gg \pm 2 \gg \pm 3$ & $\mathrm{~s}$ & rotating, timedep. \\
\hline $1-\mathrm{mRIB}_{A \leftrightarrow B}^{t}$ & $\pm 1> \pm 2> \pm 3$ & a & alternating $A \leftrightarrow B$, rotating, timedep. \\
\hline$M 1^{t}$-flow & $1 \gg 0 \gg-1$ & $\mathrm{u}$ & transitional \\
\hline $\mathrm{M}(-1)^{t}$-flow & $-1 \gg 0 \gg 1$ & $\mathrm{u}$ & transitional \\
\hline $1-\mathrm{mRIB}_{2}$ & $\pm 1 \gg \pm 2 \gg \pm 3$ & $\mathrm{~s}$ & rotating \\
\hline $1-\mathrm{mRIB}_{3}$ & $\pm 1 \gg \pm 3 \gg \pm 2$ & $\mathrm{~s}$ & rotating \\
\hline $1-\mathrm{mRIB}_{A \leftrightarrow B}$ & $\pm 1 \gg \pm 3 \gg \pm 2$ & hc & alternating $A \leftrightarrow B$, rotating \\
\hline L1R1-MCS & $1>-1$ & $\mathrm{~s}$ & left-winding, rotating \\
\hline
\end{tabular}

where $\mathbf{u}_{m}\left(\mathbf{u}_{m}^{*}\right)$ is the $m$ th (complex conjugate) Fourier mode of the velocity field. Thus, for the axisymmetric solutions $(m=0)$, e.g., CCF and TVF, only $E_{0}$ is nonzero.

\section{B. Nomenclature}

The present study focuses on flow dynamics in a relatively short periodic domain with axial wavelength $\lambda=1.6$ and counter-rotating cylinders for fixed outer Reynolds number $\operatorname{Re}_{o}=-125$. A common feature shared by most flows in the counter-rotating case in the absence of any radial flow is that they are nonaxisymmetric. Typically $m= \pm 1$ for certain parameter regime. Usually these states correspond to helical SPIs. However, in the present study most states are (m)RIB solutions which are superposition of SPIs, which means that although helical modes $m= \pm 1$ are dominant [4] their vortex structure typically has a strong azimuthal orientation. However, RIBs are axial standing waves, while they can rotate azimuthally. Thus, in a comoving frame of reference they became stationary. Table I provides an overview of various flows discussed in this work. Nomenclature, including main characteristics, dominant modes, stability, and corresponding flow dynamics are indicated.

\section{RESULTS}

\section{A. Bifurcation behavior and parameter space}

\section{Bifurcation in $R_{i}$ with constant radial flow $\alpha=12$}

Figure 2 illustrates the branches of TVF (blue circles), 1-RIB (green lozenges), and 1-mRIB ${ }^{t}$ (green lozenges) for fixed radial flow $\alpha=12$ with increasing $\operatorname{Re}_{i}$ [indicated by the arrow in the phase diagram in Fig. 4(a)]. Solid lines with closed symbols indicate stable and dashed lines with open symbols indicate unstable solutions, respectively. Shown are the modal kinetic energy $\bar{E}_{\text {kin }}$ Eq. (2.6) [Fig. 2(a)] and the dominant radial flow field amplitudes $\left|\bar{u}_{m, n}\right|$ [Fig. 2(b)] at midgap and 

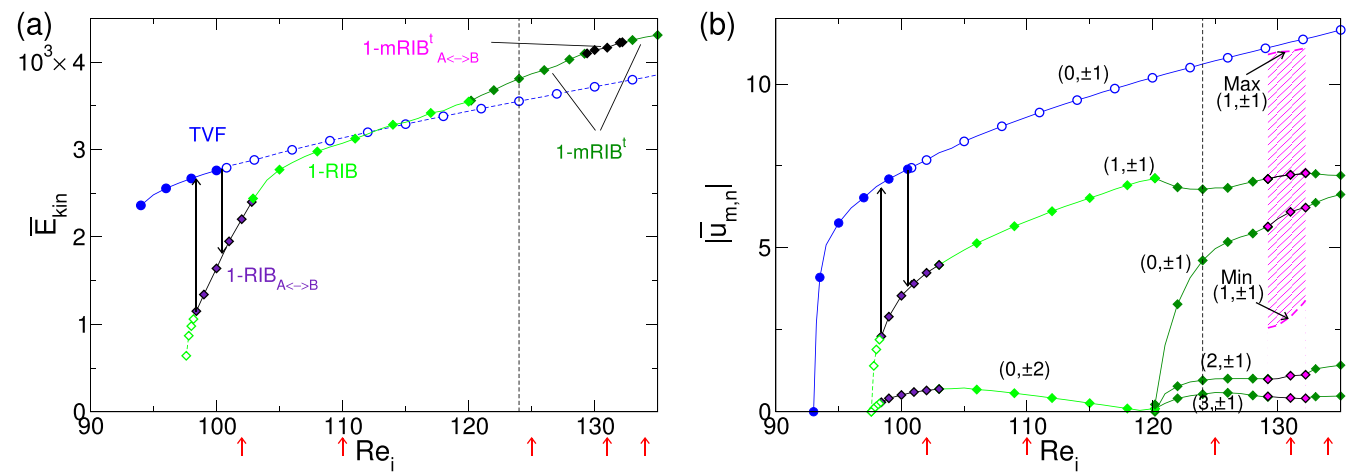

FIG. 2. Bifurcation diagrams for various vortex structures versus $\operatorname{Re}_{i}$ for $\alpha=12$. Shown are (a) (timeaveraged) modal kinetic energy $\bar{E}_{\text {kin }}(2.6)$ and (b) the dominant (time-averaged) radial flow field amplitudes $\left|\bar{u}_{m, n}\right|$ at mid-gap contributed from the modes $(m, n)$ as indicated. Solid [dashed] lines with filled [open] symbols refer to stable [unstable] solutions. Flow structures are labeled in (a); see also Table I and text for further explanation. Vertical arrows within the diagram indicate the direction of transition when one solution loses its stability in favor of another one. For comparison the energy of the CCF basic state is also shown. Small vertical arrows below the abscissa highlight parameters for which solutions are presented in more detail in the following.

midheight, respectively. Note that for time-dependent solutions the quantities are averaged over one period.

Starting at low $\operatorname{Re}_{i}$ (left in Fig. 2) and increasing $\mathrm{Re}_{i}$ the CCF basic state is succeeded by TVF that appears via a circle pitchfork bifurcation at $\mathrm{Re}_{i} \approx 92.8$ and remains stable until $\operatorname{Re}_{i} \approx 100.6$ where it loses stability to $1-\mathrm{RIB}_{A \leftrightarrow B}$. Both SPI and RIB appear via a primary Hopf bifurcation with a common, finite frequency out of $\mathrm{CCF}$ at $\mathrm{Re}_{i} \approx 97.6$, as a consequence of the breaking of the $O(2)$ symmetry (here in the axial direction) and are unstable close to onset. Eventually RIB become stabilized at $\mathrm{Re}_{i} \approx 98.4$. However, initially the RIB solution is unstable with periodic alternation between two symmetrically related $1-\mathrm{RIB}_{A}$ and $1-\mathrm{RIB}_{B}$, which generate the heteroclinic cycle (hc) $1-\mathrm{RIB}_{A \leftrightarrow B}$. Although azimuthally travelling, 1-RIB ${ }_{A}$ and $1-\mathrm{RIB}_{B}$ are stationary in a rotating frame of reference identifying them as saddles. With increasing $\mathrm{Re}_{i}$ both solutions eventually become stable at $\mathrm{Re}_{i} \approx 103.1$ and $1-\mathrm{RIB}_{A \leftrightarrow B}$ disappears. For higher $\mathrm{Re}_{i}$ they are stable, coexisting until $\mathrm{Re}_{i} \approx 120.2$ at which a new solution is born. In a second supercritical Hopf bifurcation involving larger azimuthal modes with $m= \pm 2$ and $m= \pm 3$ ), a new time-dependent mixed-ribbon solution
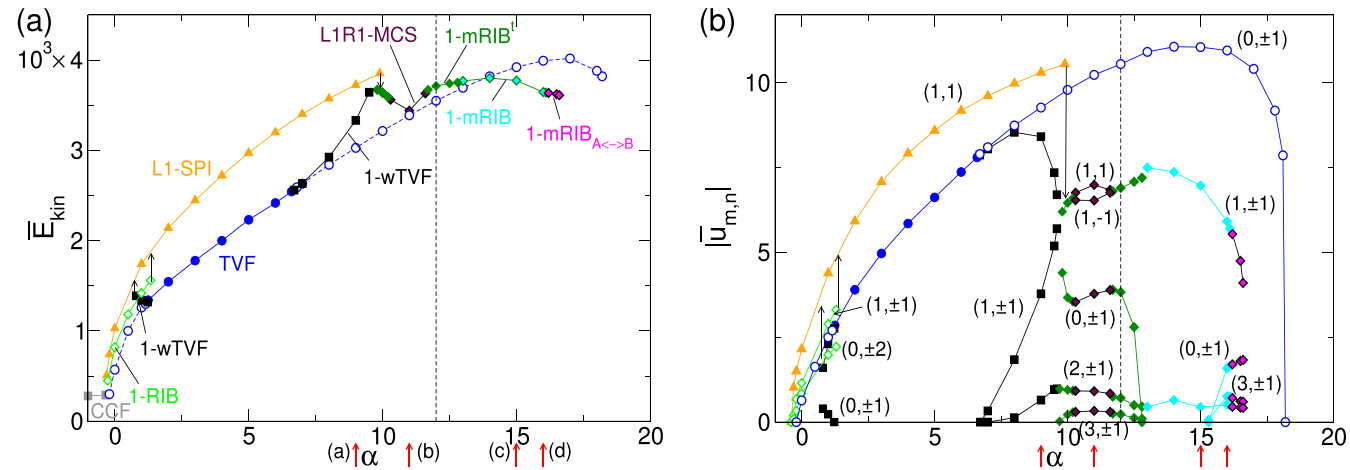

FIG. 3. Bifurcation diagrams for various vortex structures versus $\alpha$ for $\operatorname{Re}_{i}=124$. For further description see text and caption of Fig. 2. 


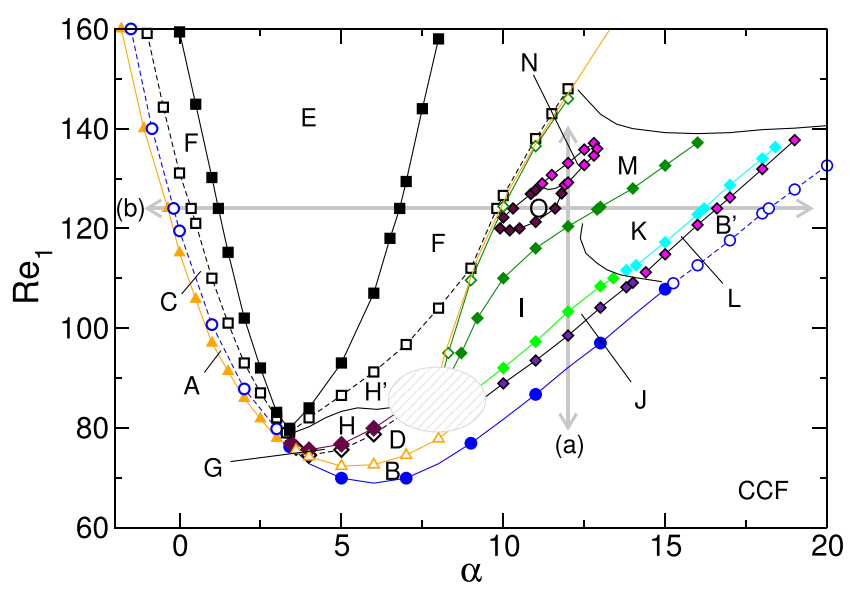

FIG. 4. $\left(\operatorname{Re}_{i}, \alpha\right)$ parameter space illustrating various (stable and unstable) solutions indicated as in Table II. The vertical and horizontal arrows (a) and (b) indicate the parameter range of the bifurcation diagrams presented in Figs. 2 and 3, respectively. In the hatched area, other perturbations, mainly with larger wave numbers, destabilize the solutions. See text for further explanation.

$\left(1-\mathrm{mRIB}^{t}\right)$ is born. Note that here the time-dependence is crucial to distinguish this from the stationary 1-mRIB solution (cf. Fig. 3) discussed later. Continuously increasing $\operatorname{Re}_{i}$ the 1 -mRIB ${ }^{t}$ exists until the end of the parameter range investigated. But in the parameter region $129.3 \lesssim \operatorname{Re}_{i} \lesssim 132.6$ the flow is again alternates between two symmetrically related solutions $1-\mathrm{mRIB}_{A}^{t}$ and $1-\mathrm{mRIB}_{B}^{t}$ and thus generates another heteroclinic cycle $1-\mathrm{mRIB}_{A \leftrightarrow B}$, similar to the scenario described previously with 1-RIB $\mathrm{RI \leftrightarrow B}_{A}$. Starting at the largest $\operatorname{Re}_{i}$ (right in Fig. 2) and continuously decreasing $\operatorname{Re}_{i}$ one finds the analogous sequence of solutions in reverse order with the only difference that at $\operatorname{Re}_{i} \approx 98.4$ the heteroclinic cycle 1-RIB $\mathrm{R}_{A \leftrightarrow B}$ and the flow evolves to the only remaining stable solution, TVF.

Regarding Fig. 2(a) it is evident that for low $\mathrm{Re}_{i}$ the TVF is more energetic than the various RIBs. But this eventually changes with increasing $\mathrm{Re}_{i}$, in particular, after TVF loses stability to 1-RIB (vertical arrow). For $\mathrm{Re}_{i} \gtrsim 115$ the various more complex 1-RIB solutions are more energetic than the pure TVF.

\section{Bifurcation in $\alpha$ with constant Reynolds number $R_{i}=124$}

After the previous discussion for constant radial flow $(\alpha=12)$, Fig. 3 presents the bifurcation scenario as a function of radial flow $\alpha$ at fixed inner Reynolds number $\operatorname{Re}_{i}=124$ (see arrow (b) in the phase diagram Fig. 4). As before the (time averaged) modal kinetic energy $\bar{E}_{\text {kin }}$ and mode amplitudes $\left|\bar{u}_{m, n}\right|$ are shown. Variation in $\alpha$ results in a more complex bifurcation scenario compared to variation with $\mathrm{Re}_{i}$.

Starting with small but negative, inwardly directed radial flow $\alpha<0$, (left in Fig. 3) one sees first the two solutions 1-SPI and 1-RIB to appear together in a supercritical, $O(2)$ symmetry-breaking Hopf bifurcation at $\alpha \approx-0.4$. While 1-SPI is stable from the beginning, 1-RIB is stabilized later with increasing radial flow at $\alpha \approx 0.1$ but loses its stability eventually again at $\alpha \approx 1.3$ where it evolves into the stable solution 1-SPI. 1-SPI remains stable up to $\alpha \approx 9.9$, where it becomes unstable and evolves to a more complex mixed cross-spiral (MCS) $[48,49]$, here L1R1-MCS solution. It should be noted that this is a stable MCS without any symmetry restrictions [48]. However, an unstable branch of TVF bifurcates at $\alpha \approx-0.2$ and the first stable toroidal solution to appear with increasing $\alpha$ is the wavy solution 1 -wTVF at $\alpha \approx 0.8$ which loses its stability shortly afterwards again at $\alpha \approx 1.3$. At this point it branches together with the hereafter stable TVF before this eventually also loses its stability at $\alpha \approx 6.7$. Here the modes $(1, \pm 1)$ become finite, generating another 1-wTVF [41]. Meanwhile, TVF continues as an unstable solution until strong radial outflow 
TABLE II. Various regions, labeled A-O, as presented in the $\left(\mathrm{Re}_{i}, \alpha\right)$ parameter space diagram (Fig. 4) including their stability properties: stable (s), unstable (u), nonexistent (-), heteroclinic cycle (hc), alternating (a).

\begin{tabular}{|c|c|c|c|c|c|c|c|c|c|c|c|c|c|c|c|}
\hline \multirow[t]{2}{*}{ Solution } & \multicolumn{15}{|c|}{ Region } \\
\hline & A & $\mathrm{B}\left[\mathrm{B}^{\prime}\right]$ & C & D & E & F & G & $\mathrm{H}\left[\mathrm{H}^{\prime}\right]$ & I & $\mathrm{J}$ & $\mathrm{K}$ & $\mathrm{L}$ & M & $\mathrm{N}$ & $\mathrm{O}$ \\
\hline TVF & - & $\mathrm{s}[\mathrm{u}]$ & $\mathrm{u}$ & $\mathrm{s}$ & $\mathrm{s}$ & $\mathrm{u}$ & $\mathrm{s}$ & $\mathrm{s}[\mathrm{u}]$ & $\mathrm{s}$ & $\mathrm{s}$ & $\mathrm{u}$ & $\mathrm{u}$ & $\mathrm{u}$ & $\mathrm{u}$ & $\mathrm{u}$ \\
\hline 1-wTVF & - & - & - & - & - & $\mathrm{s}$ & - & - & - & - & - & - & - & - & - \\
\hline SPI & $\mathrm{s}$ & - & $\mathrm{s}$ & $\mathrm{u}$ & $\mathrm{s}$ & $\mathrm{s}$ & $\mathrm{u}$ & $\mathrm{s}$ & $\mathrm{u}$ & $\mathrm{u}$ & $\mathrm{u}$ & $\mathrm{u}$ & $\mathrm{u}$ & $\mathrm{u}$ & $\mathrm{u}$ \\
\hline wSPI & - & - & - & - & - & - & $\mathrm{s}$ & - & - & - & - & - & - & - & - \\
\hline 1-RIB & $\mathrm{u}$ & - & $\mathrm{u}$ & $\mathrm{u}$ & $\mathrm{u}$ & $\mathrm{u}$ & $\mathrm{u}$ & $\mathrm{u}$ & $\mathrm{s}$ & - & - & - & - & - & - \\
\hline $1-\mathrm{RIB}_{A \leftrightarrow B}$ & - & - & - & - & - & - & - & - & - & hc & - & - & - & - & - \\
\hline $1-\mathrm{mRIB}_{A \leftrightarrow B}$ & - & - & - & - & - & - & - & - & - & - & - & hc & - & - & - \\
\hline 1-mRIB & - & - & - & - & - & - & - & - & - & - & - & - & $\mathrm{s}$ & - & - \\
\hline $1-\mathrm{mRIB}^{t}$ & - & - & - & - & - & - & - & - & - & - & - & - & - & $\mathrm{a}$ & - \\
\hline $1-\mathrm{mRIB}_{A \leftrightarrow B}^{t}$ & - & - & - & - & - & - & - & - & - & - & - & - & - & $\mathrm{a}$ & - \\
\hline L1R1-MCS & - & - & - & - & - & - & - & - & - & - & - & - & - & - & $\mathrm{s}$ \\
\hline
\end{tabular}

$\alpha \approx 18.1$ where it disappears leaving only the CCF basic state behind. While increasing $\alpha$ the contribution of the helical modes $(1, \pm 1)$ within the 1 -wTVF solution continuously increases until $\alpha \approx 9.6$. Here the toroidal 1 -wTVF disappears when it changes toward the helical time-dependent $1-\mathrm{mRIB}^{t}$. Continuously increasing $\alpha, 1-\mathrm{mRIB}^{t}$ loses stability against L1R1-MCS at $\alpha \approx 10.4$ as the two dominant mode amplitudes $(m=1$ and $m=-1)$ separate and become unequal $[48,49]$. However, MCS only exist in a narrow parameter window and for increasing $\alpha$ the flow moves back to the former 1-mRIB ${ }^{t}$ solution at $\mathrm{Re}_{i} \approx 11.6$ before this solution loses its time dependence and ends in a stationary 1 -mRIB at $\alpha \approx 12.8$. Here the toroidally dominant modes $(0, \pm 1)$ vanish [Fig. 3(b)] and leave a steady 1 -mRIB behind. With increasing $\alpha$ the 1 -mRIB exists initially as $1-\mathrm{mRIB}_{2}$ (Fig. 14) with dominant $m= \pm 2$ modes, which changes to $1-\mathrm{mRIB}_{3}$ (Fig. 15) with dominant $m= \pm 3$ modes at $\alpha \approx 15.3$. Eventually also 1 -mRIB becomes unstable generating $1-\mathrm{mRIB}_{A \leftrightarrow B}$ (similar to the scenario for $1-\mathrm{RIB}_{A \leftrightarrow B}$ ) (Fig. 2). Finally, also $1-\mathrm{mRIB}_{A \leftrightarrow B}$ ceases to exist at $\alpha \approx 16.6$ and leaving behind only the CCF basic state behind.

With decreasing $\alpha$ one finds essentially the same sequence of solutions in the opposite order as with increasing $\alpha$, starting with $1-\mathrm{mRIB}_{A \leftrightarrow B}$ and ending in 1-wTVF from which the flow evolves to 1 -SPI after losing stability at $\alpha \approx 1.45$.

\section{3. $\left(\operatorname{Re}_{i}, \alpha\right)$ parameter space}

Figure 4 illustrates the $\left(\operatorname{Re}_{i}, \alpha\right)$ parameter space investigated here and provides an overview of the solutions existing for the control parameters. The bifurcation diagrams of Figs. 2 and 3 were obtained along the two arrows labeled (a) and (b).

Blue and orange lines denote the well-known primary bifurcation thresholds out of the CCF basic state for TVF and SPI, respectively. The modifications in the stability threshold with variation in $\alpha$ has been previously reported [26,38]. Both radial inflow and strong radial outflow destabilize the system, while a moderate radial outflow (here $0<\alpha \lesssim 17.6$ ) stabilizes the system. However, variation of $\alpha$ also alters stability and sequence of primary bifurcating structures itself. For radial inflow and slight radial outflow a stable helical SPI branch bifurcates primary out of CCF, while with increasing $\alpha$ a branch of stable toroidal TVF is the first to appear. The two thresholds meet at the point of higher codimension (cf. discussion in Ref. [41]) at $\alpha \approx 3.5$ where the stability is exchanged. For $\alpha \gtrsim 15.2$ TVF bifurcates only unstable. The boundaries and curves above TVF and SPI stability thresholds separate the various regions, labeled A-O, of various flow structures, as listed in Table II including their stability properties [stable (s), alternating (a), unstable (u), nonexistent (-)]. 


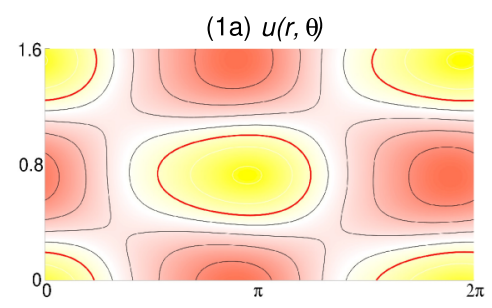

(2a)

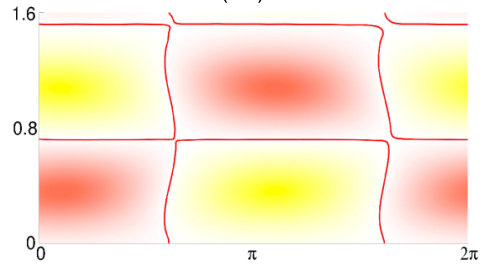

(3a)

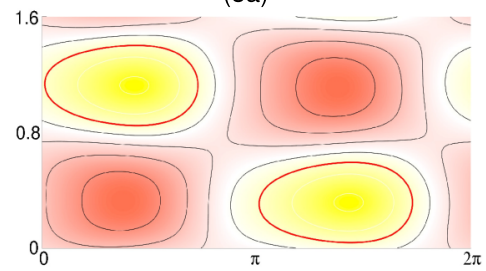

(1b) $\eta=+70$

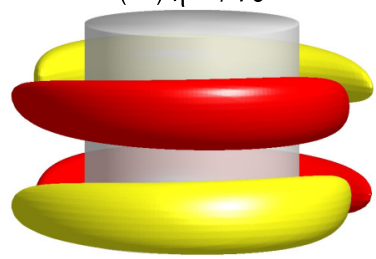

(2b)

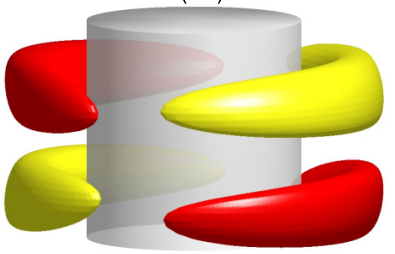

(3b)

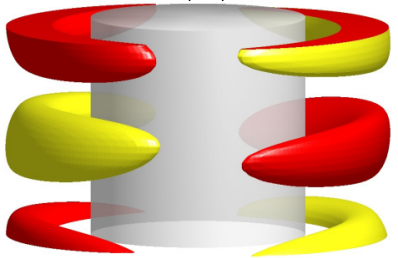

(1c) $[u(r, z), w(r, z)]$

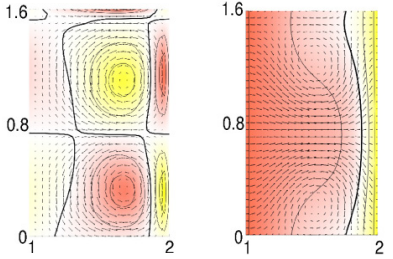

(2c)
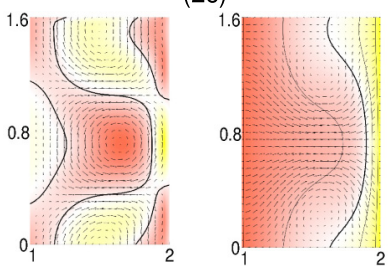

(3c)
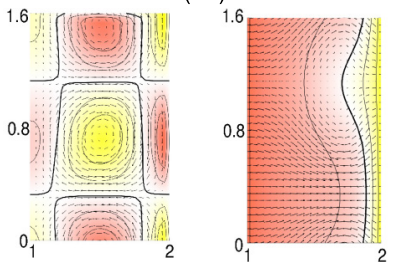

FIG. 5. Flow visualization of $1-\mathrm{RIB}_{A \leftrightarrow B}$. Visualization of (1) $1-\mathrm{RIB}_{A}$, (2) transitional M0-flow and (3) $1-\mathrm{RIB}_{B}$. Shown are (a) the radial velocity $u(\theta, z)$ on an unrolled cylindrical surface in the annulus at mid-gap [red (yellow) color indicates in (out) flow], (b) isosurfaces of $\eta$ [red (dark gray) and yellow (light gray) colors correspond to positive and negative values, respectively, with zero specified as white], and (c) vector plot $[u(r, z), w(r, z)]$ of the radial and axial velocity components (including the azimuthal vorticity $\eta$ (left) and azimuthal velocity $v$ (right), respectively. The thick contour lines correspond to $\eta=0$ (left) and $v / \operatorname{Re}_{i}=0.5$ (right), respectively. Control parameters: $\alpha=12, \operatorname{Re}_{i}=102$. Note, for the M0-flow $\eta= \pm 1.5(2 b)$.

Of special interest are the three regions in which various $(\mathrm{m})$ RIB are stable, unstable, stationary or time-dependent (cf. Table I): J: 1-RIB $\operatorname{R\leftrightarrow B}_{A \leftrightarrow B}$ L: 1-RIB ${ }_{A \leftrightarrow B}^{t} ; \mathrm{N}: 1-\mathrm{mRIB}_{A \leftrightarrow B}^{t}$;

However, we were not able to trace out the boundaries between all solutions for the full set of parameters; the hatched area in Fig. 4 indicates schematically that in this area perturbations (mainly with larger wave numbers) prematurely destabilize the solutions discussed here against other significantly more complex flow structures.

\section{B. Flow dynamics, structures and heteroclinic cycles}

In the following, we will focus on the regions containing $(\mathrm{m}) \mathrm{RIB}_{A \leftrightarrow B}^{(t)}$, i.e., alternation between the two saddles $(\mathrm{m}) \mathrm{RIB}_{A}^{(t)}$ and $(\mathrm{m}) \mathrm{RIB}_{B}^{(t)}$.

\section{Stationary ribbon: $1-R I B$}

We will start with a 1-RIB solution, which appears in heteroclinic cycles (m) $\operatorname{RIB}_{A \leftrightarrow B}^{(t)}$ between the two unstable saddles 1-RIB ${ }_{A}$ and 1-RIB ${ }_{B}$. (cf. regions $\mathrm{J}$ and $\mathrm{I}$ in phase diagram in Fig. 4). Visualizations of the two saddles 1-RIB ${ }_{A}$ and 1-RIB $B$ are presented in Fig. 5 together with the transitional, toroidal M0-flow. Regarding the radial velocity $u(\theta, z)$ on an unrolled cylindrical surface in the annulus at mid-gap [Fig. 5(a)] one can identify that $1-\mathrm{RIB}_{A}$ and $1-\mathrm{RIB}_{B}$, are shifted by $\lambda / 4$ in the axial direction. The transitional M0-flow, although quite similar to the 1-RIB, can be identified by 
the chessboard pattern in the contours of radial velocity $u(\theta, z)$ [Fig. $5(2 \mathrm{a})]$. The similarity results from the fact that both 1-RIB and M0-flow are axial nonpropagating structures, although their generation process is crucially different. 1-RIB is a nonlinear superposition of two counter-rotating and axially propagating, left- and right-winding, helical SPIs with equal mode amplitudes [4]. Thus, 1-RIB is a standing wave in axial direction while rotating in azimuth. Consequently, the corresponding Fourier spectrum also contain helical modes. In contrast, the spectrum for M0-flow does not contain helical modes [cf. Figs. 6(c) and 6(d)].

Figure 6 shows longer time series illustrating the time-periodic change from 1-RIB ${ }_{A}$ via transitional M0-flow toward $1-\mathrm{RIB}_{B}$ and vice versa. Illustrated are (a) global modal kinetic energy $E_{\text {kin }}$ together with local measures of the velocities $u_{1}, u_{2}$, and $u_{3}$ [radial velocity at three distinct points in the annulus: $\left.u_{1}=u(d / 2,0, \Gamma / 4), u_{2}=u(d / 2,0, \Gamma / 2), u_{3}=u(d / 2,0,3 \Gamma / 4)\right]$ and (b) dominant mode amplitudes $\left|u_{m, n}\right|$. Both $1-\mathrm{RIB}_{A}$ and $1-\mathrm{RIB}_{B}$ have identical kinetic energy, $E_{\mathrm{kin}}$, and Fourier spectra [Fig. 6(c)]. Within the transitional M0-flow $E_{\text {kin }}$ almost vanishes. Analogously, the time series of $\left|u_{m, n}\right|$ illustrate the elimination of all helical modes $m \geqslant 1$ within the M0-flow, leaving only the toroidal $m=0$ mode [Figs. 6(b) and 6(d)].

However, we emphasize that although the M0-flow only contains $m=0$ modes, it is crucially different from classical TVF, which consists of a toroidal closed vortex. For our parameters TVF branches super-critically and is stable, coexisting with other solutions (see Figs. 2 and 4). The M0flow does not contain toroidal closed vortices and additionally it is not axisymmetric [see Fig. 5(2a)]. Instead, M0-flow retains the symmetries of RIB, which are given by the symmetry group $Z_{2}$.

The main dynamics during the transition from $1-\mathrm{RIB}_{A}$ toward $1-\mathrm{RIB}_{B}$ can be described as an annihilation and regeneration of a vortex pairs (in the axial direction). First the vortex pairs of $1-\mathrm{RIB}_{A}$ relocate in its axial position (direction), while moving closer together which results in stretch and compression in the annulus. With increasing time the compression of the vortex pairs eventually results in an annihilation of these vortices, while at the same time two new vortices are generated, which then expand into the annulus. The newly formed vortex pair is shifted about $\lambda / 4$ in axial direction and grows to establish again a temporal solution $1-\mathrm{RIB}_{B}$. Hereafter the system remains for a time that depends on the parameters in $1-\mathrm{RIB}_{B}$, before eventually the scenario restarts with a similar back switch to $1-\mathrm{RIB}_{A}$. The process repeats as long no other external parameters, e.g., $\alpha$ or $\mathrm{Re}_{i}$ are changed. Heteroclinic connections between different solutions are not uncommon and has been studied in various systems [50-55,59] in the past. For the current work of special interest is the recent observation for RIB solutions [60] with oscillatory and nonoscillatory transition.

The phase portraits Figs. $6(\mathrm{e})-6(\mathrm{~h})$ spanned by $u_{1}, u_{2}$, and $u_{3}$ illustrate the connections, i.e., limit cycles, of $1-\mathrm{RIB}_{A}$ and $1-\mathrm{RIB}_{B}$. The color coding corresponds to the time series presented in Figs. 6(a) and 6(b). The local quantities $u_{1}$ and $u_{2}$ versus $u_{3}$ highlight a perpendicular orientation for $1-\mathrm{RIB}_{A}$ and $1-\mathrm{RIB}_{B}$, respectively, resulting in a clover-leaf shape in the corresponding phase portraits [Figs. 6(e)-6(h)], with the transitional M0-flow in the core region at the clover-leaf intersection. The dynamics of the local quantities within $1-\mathrm{RIB}_{A}$ and $1-\mathrm{RIB}_{B}$ can also be detected in the time series of $u_{1}\left[u_{2}\right]$ in Fig. 6(a). In fact $u_{1}$ and $u_{2}$ just behave contrarily in $1-\mathrm{RIB}_{A}$ and $1-\mathrm{RIB}_{B}$, respectively. While $u_{1}$ has small modulation amplitudes in $1-\mathrm{RIB}_{A}$ and large ones in $1-\mathrm{RIB}{ }_{B}$, the opposite holds for $u_{2}$.

Figure 7 provides a comparison between ribbon solutions, stable and unstable. Shown are azimuthal velocity contour profiles $v / \operatorname{Re}_{i}=0.5$ within the gap (azimuthal position as indicated). Figures 7(b) and 7(c) present profiles of $1-\mathrm{RIB}_{A}$ and $1-\mathrm{RIB}_{B}$ at $\mathrm{Re}_{i}=102$, at which $1-\mathrm{RIB}_{A \leftrightarrow B}$ is unstable with alternation between both. For comparison a stable 1-RIB solution $\left(\operatorname{Re}_{i}=110\right)$ is also presented indicating differences in the contours based on the azimuthal position. While in $1-\mathrm{RIB}_{A \leftrightarrow B}$ the contours for $\theta=0$ (black) and $\theta=3 / 4 \pi$ (blue) as well as for $\theta=\pi / 2$ (red) and $\theta=\pi$ (green) have similar amplitudes, they differ strongly for 1-RIB.

In the heteroclinic cycle the flow remains in one of the saddles, $1-\mathrm{RIB}_{A}$ or $1-\mathrm{RIB}_{B}$, for almost identical time $t \approx 3.8$ [cf. Figs. 6(a) and 6(b)]. The persistence time only changes with modification in external parameters, e.g., $\alpha$ or $\mathrm{Re}_{i}$. Long time simulations confirmed this observation of constant persistence time within one of the solutions as well as the transition time between them. For 1-RIB $A$ 


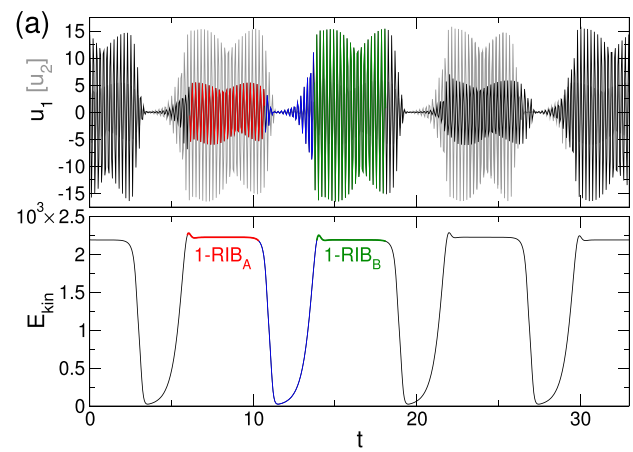

(c)
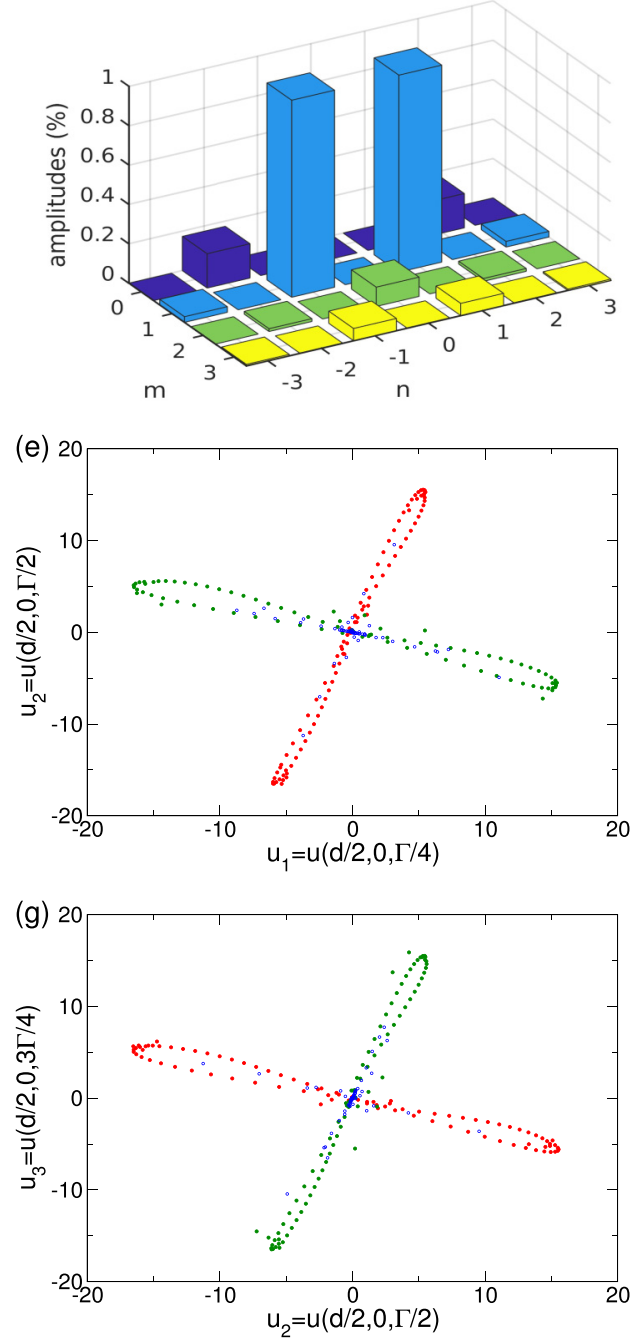

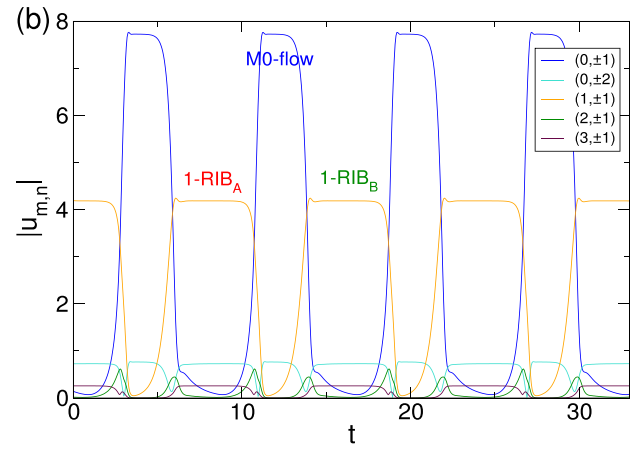

(d)
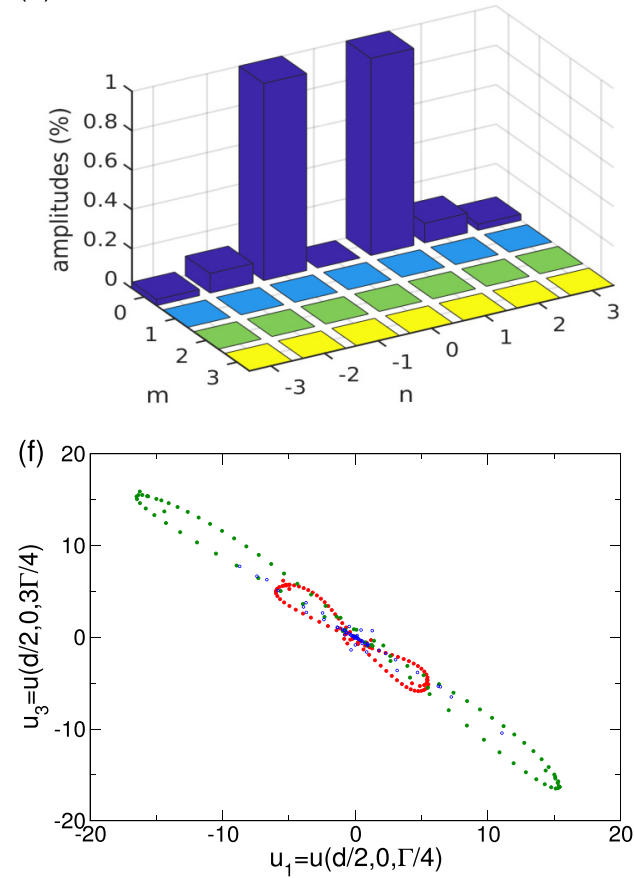

(h)

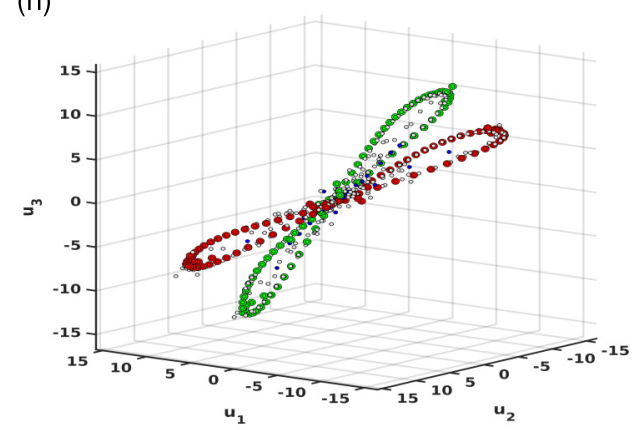

FIG. 6. Visualization of $1-\operatorname{RIB}_{A \leftrightarrow B}$. Shown are dynamics with time of (a) $E_{\text {kin }}$ and $u_{1}$ [ $u_{2}$ (light gray)], (b) modes $\left|u_{m, n}\right|$; Fourier spectrum ( $m, n$ ) of (c) 1-RIB A $_{A, B}$ and (d) M0-flow, respectively. (e)-(h) Phase portraits spanned by $u_{1}, u_{2}$, and $u_{3}$ (see text for further description). Control parameters: $\alpha=12, \operatorname{Re}_{i}=102$. For all but $(\mathrm{c}, \mathrm{d})$ red and green colors indicate the $1-\mathrm{RIB}_{A}$ and $1-\mathrm{RIB}_{B}$, respectively, while blue colors indicate the transitional M0-flow. 


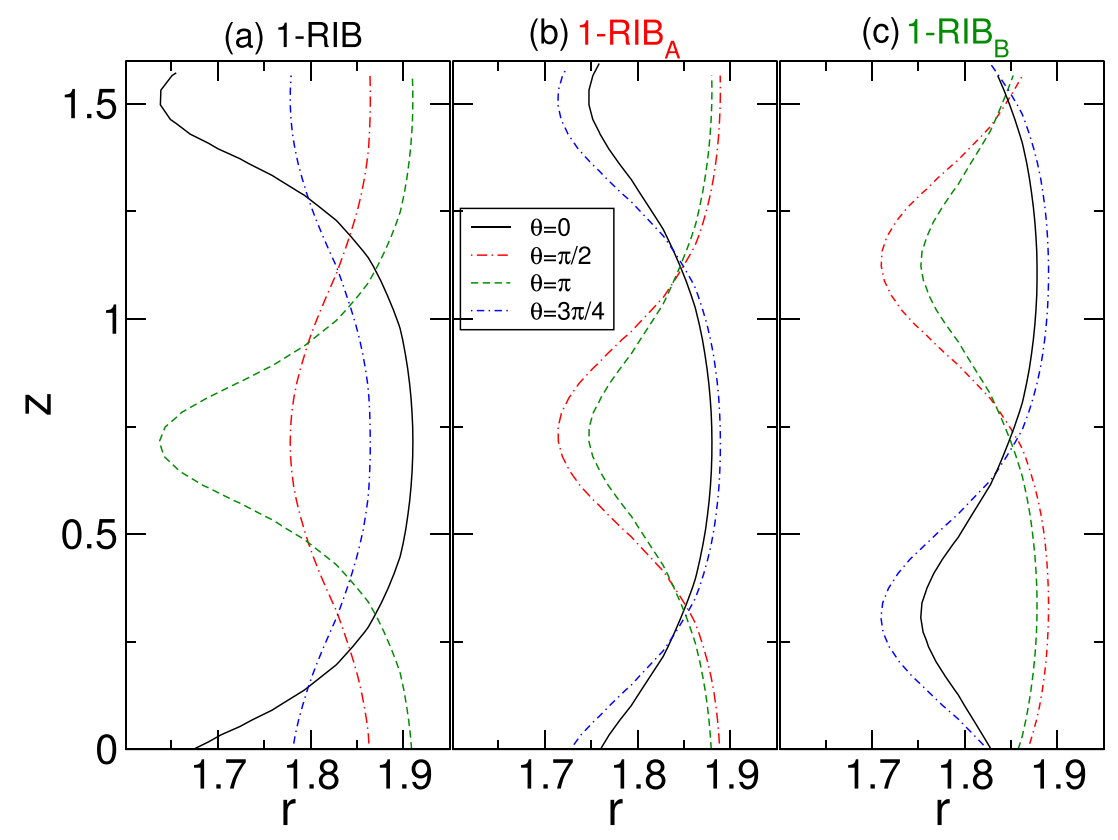

FIG. 7. Azimuthal velocity contour profiles at $v / \operatorname{Re}_{i}=0.5$ in bulk gap with variation for various 1-RIB (a) stable 1-RIB at $\mathrm{Re}_{i}=110$, Snapshots within $1-\mathrm{RIB}_{A \leftrightarrow B}$ for (b) $1-\mathrm{RIB}_{A}$ and (c) $1-\mathrm{RIB}_{B}$ at $\mathrm{Re}_{i}=102$ and $\alpha=12$.

and 1-RIB ${ }_{B}$ the persistence time increases with increasing $\mathrm{Re}_{i}$ and thus approaching the stable 1-RIB at $\operatorname{Re}_{i}=103.1$ (Fig. 2).

\section{Time-dependent Mixed-Ribbon: 1-mRIB ${ }^{t}$}

In what follows we will focus on the various mRIB, which present the next step in complexity after the classical RIB discussed above. To our knowledge this is also the first time that mRIBs have been detected as stable solutions without any symmetry restrictions $[48,49]$. The crucial difference with 1-RIB is the fact that for 1-mRIB aside from the helical dominant modes $m= \pm 1$ additional modes $m \neq 1$ are present, which are not higher harmonics of the basic mode. This includes toroidal $m=0$ as well as other helical $m \neq 0$ modes of significantly larger amplitudes [Fig. 8(c)] (Just the opposite holds for 1-wTVF [41]). Additionally, the nonlinear driven modes $(m, n)=(0, \pm 2)$ is significant larger in mRIB than in WTVF due to the emergence. This results from the fact that 1 -mRIB is a superposition of oppositely winding 1 -wSPIs $[( \pm 1, \pm 1)+(\mp 1, \pm 1)=(0, \pm 2)]$, while a classical 1-RIB is a superposition of 1-SPIs.

a. Stable 1-mRIB ${ }^{t}$. Consider the stable time-dependent 1-mRIB ${ }^{t}$ at $\alpha=12, \operatorname{Re}_{i}=125$ (Figs. 8 and 9). Various time series for $1-\mathrm{mRIB}^{t}$ are shown in Fig. 8. While the global quantity $E_{\text {kin }}$ has period $\tau \approx 0.34$ (also visible in the mode amplitudes $\left|u_{m, n}\right|$ ) the velocities $u_{i}, i=\{1,2,3\}$ (as local quantities) have more complex time dynamics. Both, the spectra $(m, n)$ and time series of $\left|u_{m, n}\right|$ show that the dominant modulation mode for $1-\mathrm{mRIB}^{t}$ is $m= \pm 2$ and the corresponding $\left(u_{1}, u_{3}\right)$ phase portrait supports that $1-\mathrm{mRIB}^{t}$ lives on a two-torus shown in Fig. 8(d) [with the inset showing the Poincaré section $\left.\left(u_{3}, E_{\text {kin }}\right)\right]$. Thus, the dimensionality is one greater than that of the heteroclinic cycle shown in Fig. 6(e)-6(h). The modulation modes $m= \pm 2$ are significantly smaller than the dominant $m= \pm 1$ modes for $1-\mathrm{mRIB}^{t}$ [Fig. 8(b) and 8(c)] and only vary in a narrow range in their amplitudes over one period. Interestingly this does not apply to the toroidal mode $m=0$, which 

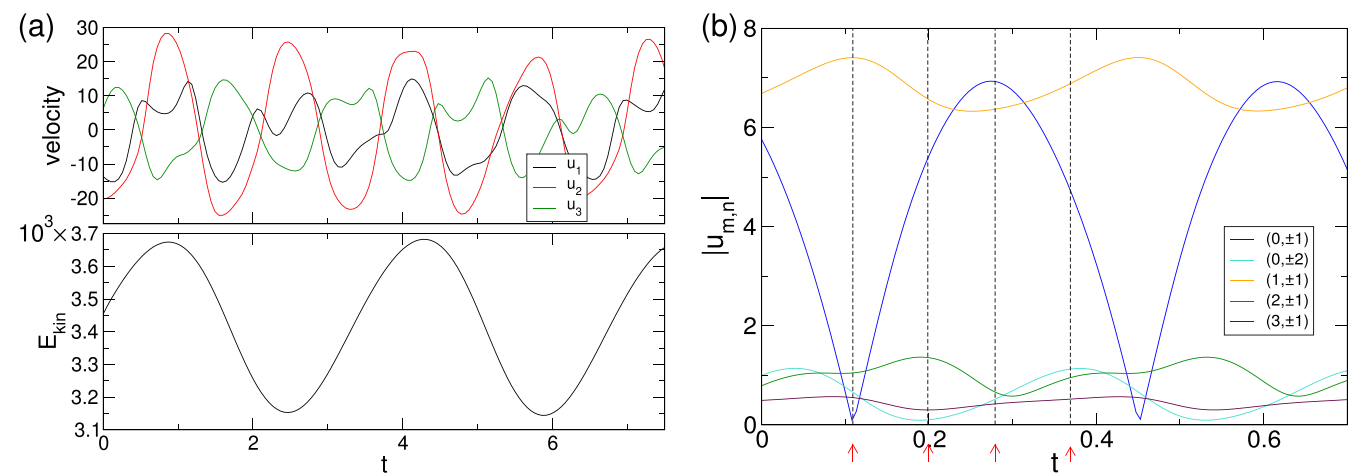

(c)
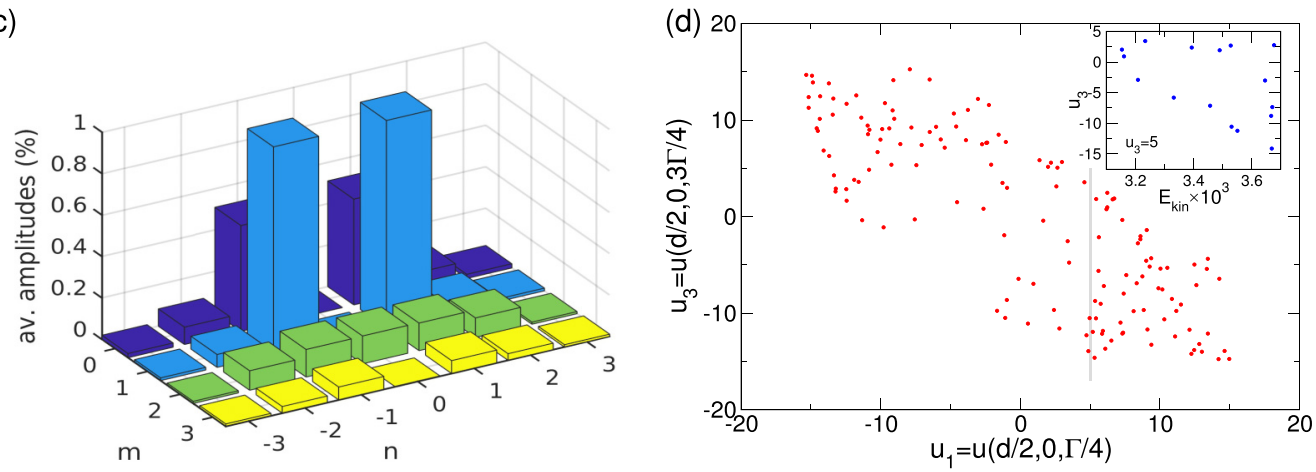

FIG. 8. Specification of $1-\mathrm{mRIB}^{t}$. Shown are dynamics with time of (a) $E_{\text {kin }}$ and velocities $u_{1}, u_{2}, u_{3}$, (b) mode amplitudes $\left|u_{m, n}\right|$; (c) time-averaged (over one period) and scaled Fourier spectrum $(m, n)$ [cf. Fig. 9(d)], and (d) phase portraits spanned by $u_{1}$ and $u_{3}$ (cf. Fig. 6). The inset shows the corresponding Poincaré section $\left(u_{3}, E_{\mathrm{kin}}\right)$ for $u_{1}=5$ (vertical gray line). Control parameters: $\alpha=12, \operatorname{Re}_{i}=125$. Small vertical arrows below the abscissa in (b) indicate time steps for which solutions are presented over one period in Fig. 9. Note the dominant modulation mode is $m= \pm 2$.

shows a huge variation within one period $\tau$. In fact, it varies between near-zero values and being the largest mode amplitude [cf. Figs. 8(b) and 9(1d)].

Figure 9 shows snapshots of $1-\mathrm{RIB}^{t}$ over one period $\tau=0.34$ [marked by vertical dashed lines and red arrows on the abscissa in Fig. 8(b)]. The dominant modulation modes $m= \pm 2$ within the $1-\mathrm{mRIB}^{t}$ can be seen in the contours of the radial velocity $u(\theta, z)$ [Figs. 9(1,2)]. The toroidal structure appearing within one period is also visible in $u(\theta, z)$ [Figs. $9(3,4)$ ], which results from the temporal dominant contribution of the $m=0$ mode in the flow structure [Fig. 9(d)].

For 1-mRIB the amplitudes of larger azimuthal wave numbers $m>1$ are equal, $\left|u_{m, n}\right|=\left|u_{-m, n}\right|$. This distinguishes mRIB from other helical superimposed flow states, e.g., MCSs [48,49], which have different dominant mode contributions $m \neq-m$.

b. Unstable 1-mRIB ${ }_{A \leftrightarrow B}^{t}$. Similar to previously discussed $1-\mathrm{RIB}_{A \leftrightarrow B}, 1-\mathrm{mRIB}_{A \leftrightarrow B}^{t}$ can also be alternating (cf. Fig. 2 and region $\mathrm{N}$ in Fig. 4).

To provide a better understanding of the transition between the alternating solutions, Fig. 10 presents the same quantities as Fig. 6. Due to time-dependence of 1-mRIB ${ }_{A \leftrightarrow B}^{t}$, the corresponding time series of local and global measures are more complex than for the stationary case of $1-\mathrm{RIB}_{A \leftrightarrow B}$. However, there are fundamental differences with the previous case.

Crucial first observation is that the transition between $1-\mathrm{mRIB}_{A}^{t}$ and $1-\mathrm{mRIB}_{B}^{t}$ can appear in two different ways, either via $\mathrm{M}^{t}$ or $\mathrm{M}(-1)^{t}$ [Fig. 10(b)], respectively. These are both helical solutions but differ in their chirality, i.e., the sign of the dominant mode $m=1$ [Fig. 10(e)] and $m=-1$ 


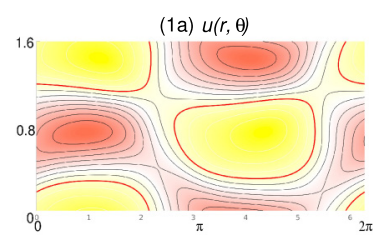

(2a)

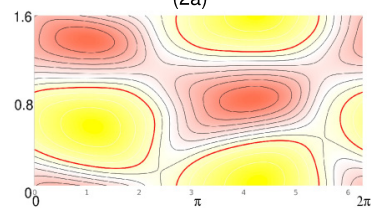

(3a)

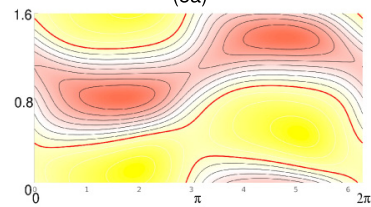

(4a)

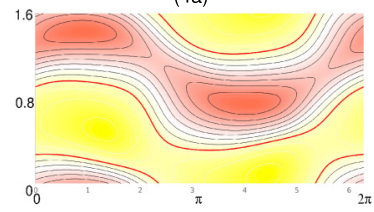

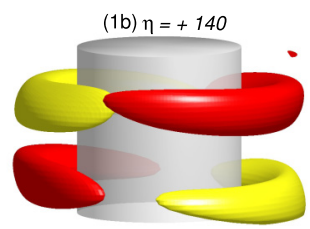

(2b)

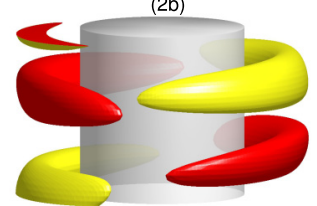

(3b)

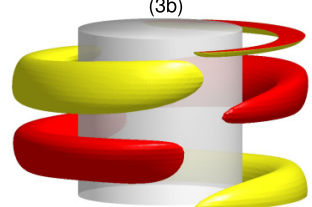

(4b)

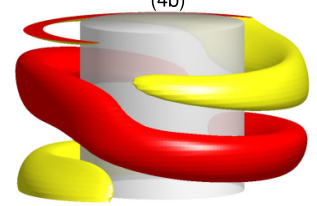

(1c) $[u(r, z), w(r, z)]$

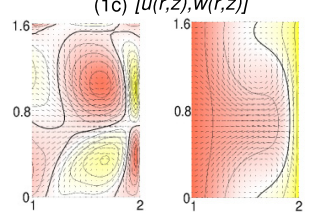

(2c)

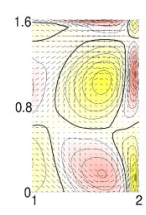

(3c)

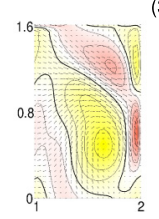

c)

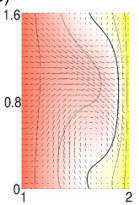

(4c)

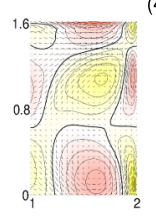

(1d)

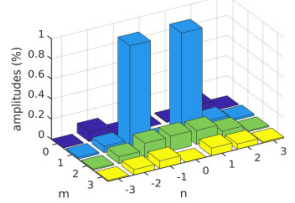

(2d)

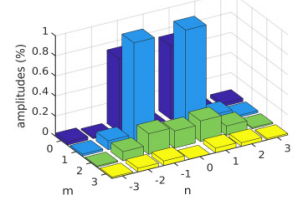

(3d)

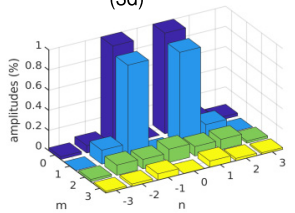

(4d)

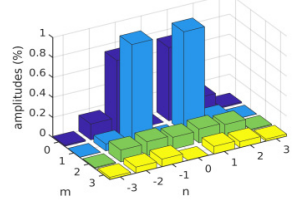

FIG. 9. Flow visualization of $1-\mathrm{mRIB}_{A \leftrightarrow B}^{t}$ over one period $\tau \approx 0.34$. Snapshots of $1-\mathrm{mRIB}^{t}$ at (1) $t=0$, (2) $t=T / 4$, (3) $t=T / 2$, and (4) $t=3 T / 4$ (cf. Fig. 8). Shown are (a) the radial velocity $u(\theta, z$ ) on an unrolled cylindrical surface in the annulus at mid-gap [red (yellow) color indicates in (out) flow], (b) isosurfaces of $\eta$ [red (dark gray) and yellow (light gray) colors correspond to positive and negative values, respectively, with zero specified as white], and (c) vector plot $[u(r, z), w(r, z)]$ of the radial and axial velocity components (including the color-coded azimuthal vorticity $\eta$ (left) and azimuthal velocity $v$ (right), respectively. The thick contour lines in (c) correspond to $\eta=0$ (left) and $v / \operatorname{Re}_{i}=0.5$ (right), respectively. (d) Corresponding spectrum $(m, n)$. Control parameters: $\alpha=12, \operatorname{Re}_{i}=125$.

[Fig. 10(g)], respectively. In the scenario discussed above for 1-RIB $\mathrm{R}_{A \leftrightarrow B}$ we always found the same transitional M0-flow independent of the direction [Fig. 5(2)]. For mRIB, the toroidal mode $m=0$ only plays a minor role [cf. Figs. 10(c)]. All flows observed within 1-mRIB ${ }_{A \leftrightarrow B}^{t}, 1-\mathrm{mRIB}_{A}^{t}, 1-\mathrm{mRIB}_{B}^{t}$ and the two transitional flows, $\mathrm{M} 1^{t}$-flow or $\mathrm{M}(-1)^{t}$-flow, are time-dependent.

Second difference from the previously discussed $1-\mathrm{RIB}_{A \leftrightarrow B}$ is the variation both in persistence time within $1-\mathrm{mRIB}_{A}^{t}$ or $1-\mathrm{mRIB}_{B}^{t}$ as well as in the transition time between the saddles. Here, both appear to be more random and we could not observe any correlation between the transitional flows and the direction of transition. The heteroclinic connection and therefore the transition from $1-\mathrm{mRIB}_{A}^{t}$ to $1-\mathrm{mRIB}_{B}^{t}$ can appear via $\mathrm{M} 1^{t}$-flow or $\mathrm{M}(-1)^{t}$-flow and vice versa [Figs. 10(b) and 11(a)]. Numerous long-time simulations did not allow us to identify any correlation between $1-\mathrm{mRIB}_{A}^{t}$ and $1-\mathrm{mRIB}_{B}^{t}$ and the direction over which intermediate flow the transition appears. An example of a long-time series is presented in Fig. 11(a) for $\left|u_{m, n}\right|$ showing numerous switches between $1-\mathrm{mRIB}_{A}^{t}$ and $1-\mathrm{mRIB}_{B}^{t}$ via $\mathrm{M}^{t}$ and $\mathrm{M}(-1)^{t}$. Although there is no special selected persistence time, it seems that for a set of system parameters there is an upper time limit, i.e., a maximum persistence time, $\tau_{p, \max }$, for the flow within a solution. Latest at this time transition will appear. For the parameters in Figs. 10, 11, and 13 we found an upper limit of the persistence time $13.7 \lesssim \tau_{p, \max } \lesssim 13.9$. The persistence time depends on the system parameters as seen for 

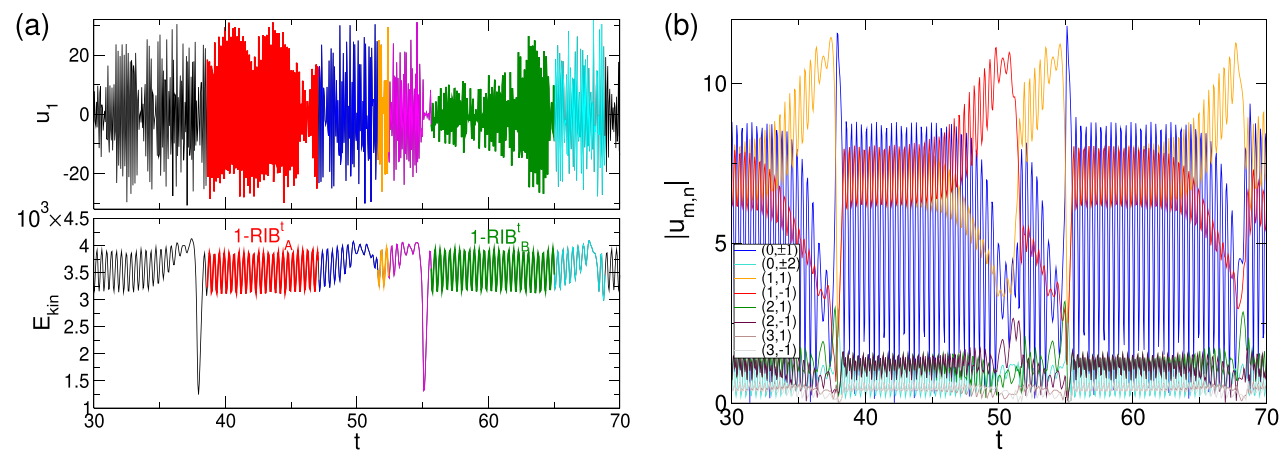

(c)
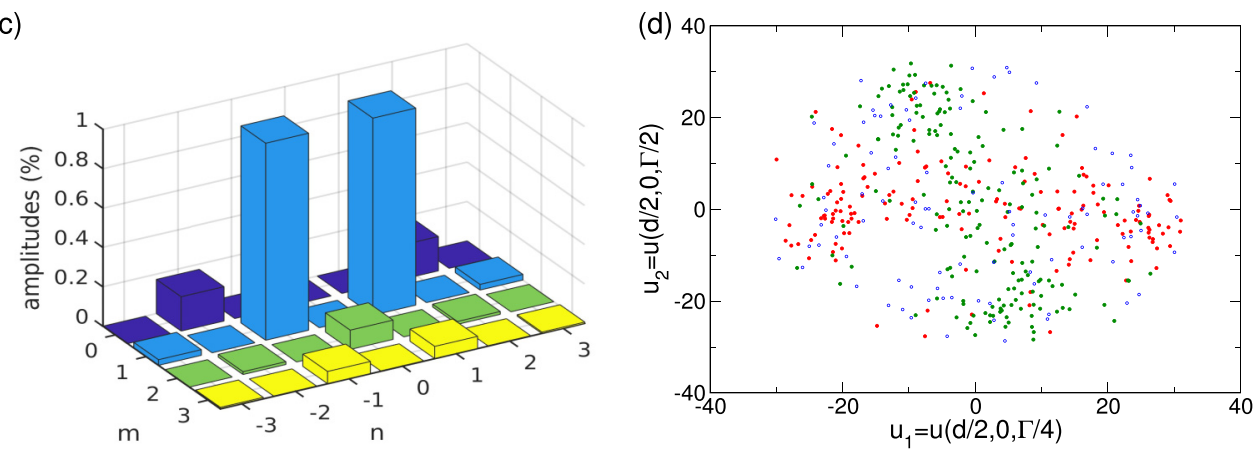

(e)
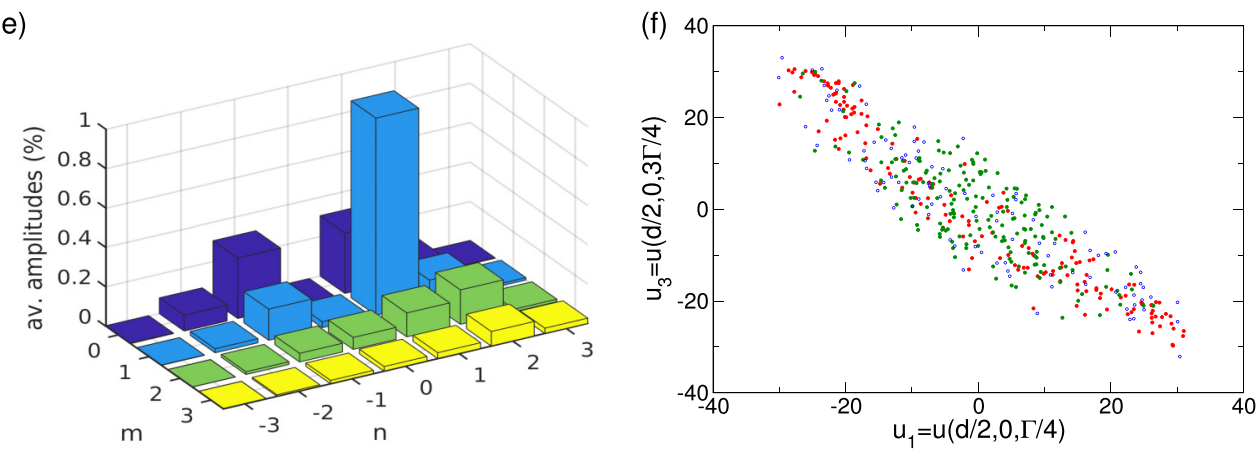

(g)

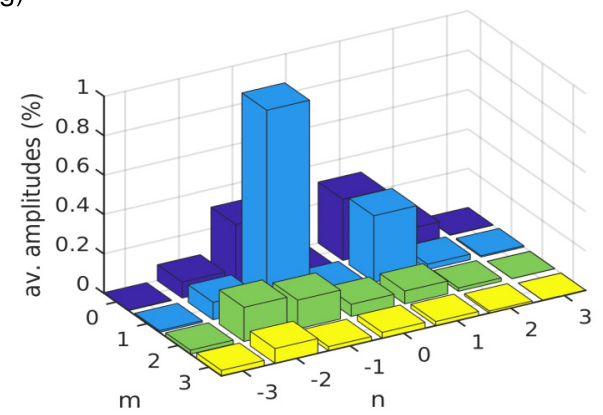

(h)

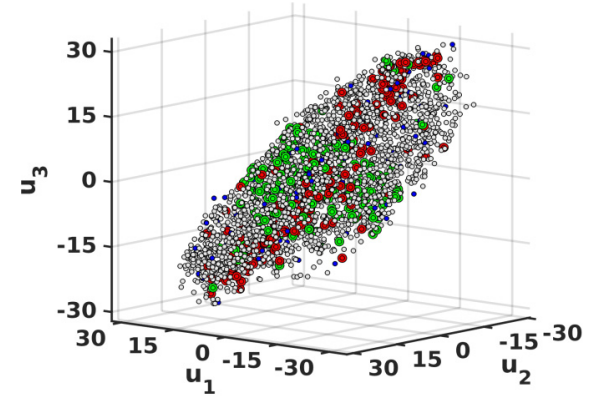

FIG. 10. Visualization of $1-\mathrm{mRIB}_{A \leftrightarrow B}^{t}$. Shown are dynamics with time of (a) $E_{\mathrm{kin}}$ and $u_{1}$, (b) modes $\left|u_{m, n}\right|$; time averaged spectra $(m, n)$ of (c) $1-\mathrm{mRIB}_{A, B}^{t}$, (e) $\mathrm{M} 1^{t}$-flow, and (g) $\mathrm{M}(-1)^{t}$-flow, respectively. (d, f, h) show corresponding phase portraits spanned by $u_{1}, u_{2}$ and $u_{3}$. Control parameters: $\alpha=12, \operatorname{Re}_{i}=131$. For all but (c, $\mathrm{e}, \mathrm{g}$ ) red and green colors indicate the solutions $1-\mathrm{mRIB}_{A}^{t}$ and $1-\mathrm{mRIB}_{B}^{t}$, respectively, while blue and magenta color highlights the transitional $\mathrm{M} 1^{t}$-flow and $\mathrm{M}(-1)^{t}$-flow. 


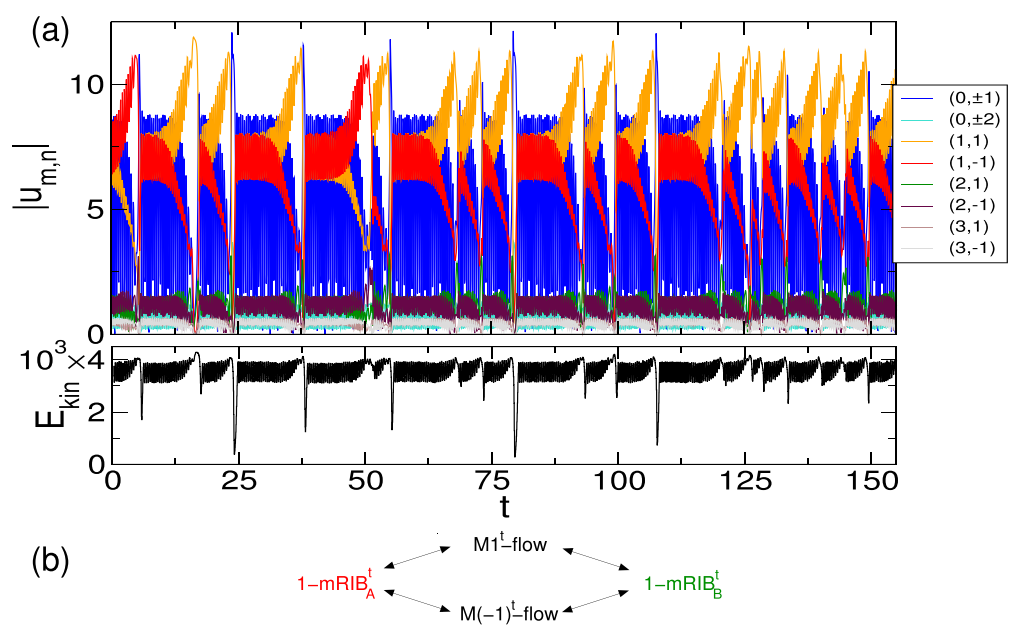

FIG. 11. (a) Time series of mode amplitude $\left|u_{m, n}\right|$ and kinetic energy $E_{\text {kin }}$ illustrating an intermittency scenario for the transition between the saddles $1-\mathrm{mRIB}_{A}^{t}$ and $1-\mathrm{mRIB}_{B}^{t}$, via $\mathrm{M} 1^{t}$-flow and $\mathrm{M}(-1)^{t}$-flow, respectively. Control parameters are the same as in Fig. 10. Note that the time series suggest a maximum persistence time. (b) Schematic illustration of the different transition behavior between 1-mRIB ${ }_{A}$ and $1-\mathrm{mRIB}_{B}$ via $\mathrm{M1}^{t}$-flow or $\mathrm{M}(-1)^{t}$-flow. The transition takes place after a random time, either via $\mathrm{M}^{t}$-flow or $\mathrm{M}(-1)^{t}$-flow.

$1-\mathrm{RIB}_{A \leftrightarrow B}$. The closer to the onset of the branching of $1-\mathrm{mRIB}_{A \leftrightarrow B}^{t}$, the larger is $\tau_{p \text {,max }}$. This is a manifestation of a complex underlying manifold with various attracting solutions in a quite narrow region. The heteroclinic connection between $1-\mathrm{mRIB}_{A}^{t}$ and $1-\mathrm{mRIB}_{B}^{t}$ seems to be of oscillatory type

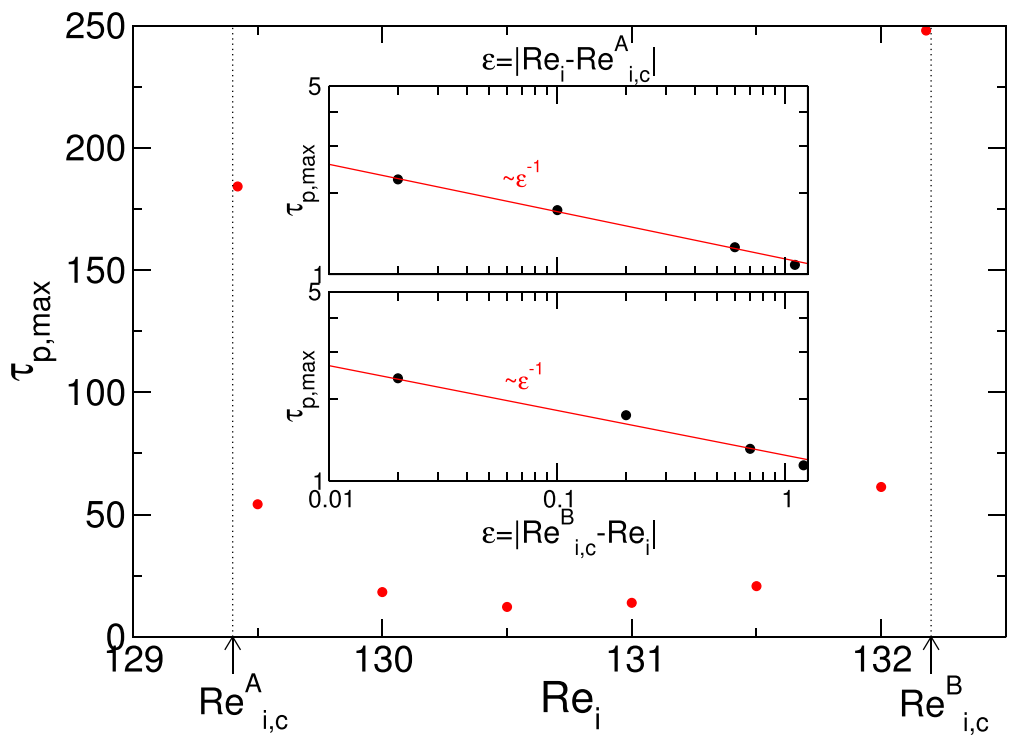

FIG. 12. Intermittency of $1-\mathrm{mRIB}_{A \leftrightarrow B}^{t}$. Variation with $\mathrm{Re}_{i}$ for the maximum persistence time $\tau_{p \text {,max }}$ of the heteroclinic cycle 1-mRIB ${ }_{A \leftrightarrow B}^{t}$ for $\alpha=12$ (cf. Fig. 2). Insets show the scaling of the $\tau_{p \text {,max }}$ with control parameter $\epsilon=\left|\operatorname{Re}_{i}-\operatorname{Re}_{i, c}^{A}\right|$ (top panel) and $\epsilon=\left|\operatorname{Re}_{i, c}^{B}-\operatorname{Re}_{i}\right|$ (bottom panel), where $\operatorname{Re}_{i, c}^{A}$ and $\operatorname{Re}_{i, c}^{B}$ are the critical values at which intermittency appears. Both scenarios suggest a type III intermittency $[61,62]$ approximated by $\propto \epsilon^{-1}$. 
(1a) $u(r, \theta)$

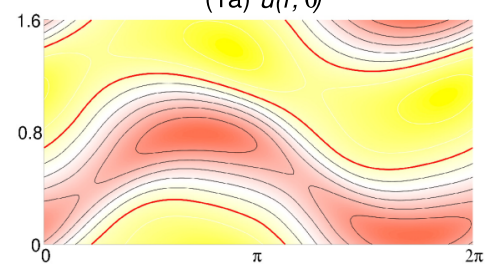

(2a)

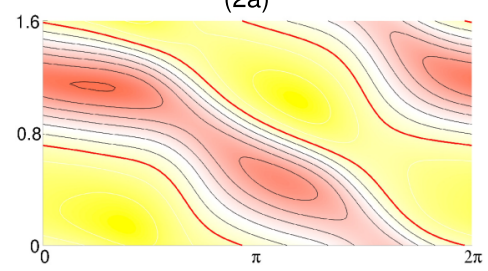

(3a)

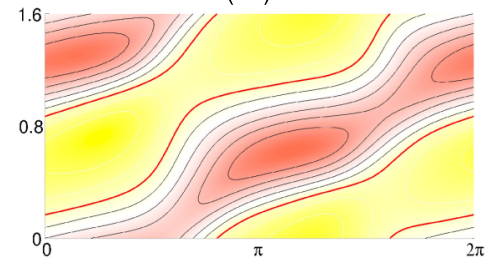

(4a)

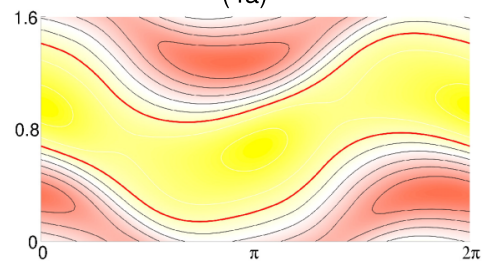

(1b) $\eta=+140$

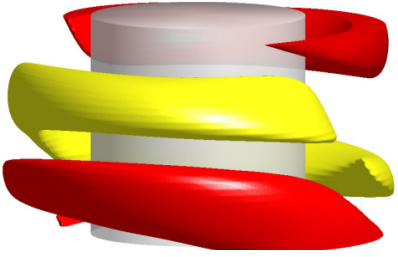

(2b)

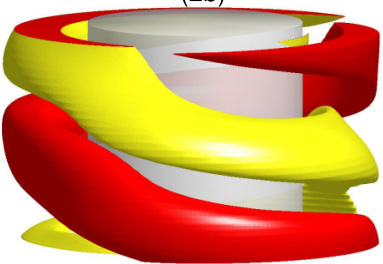

(3b)

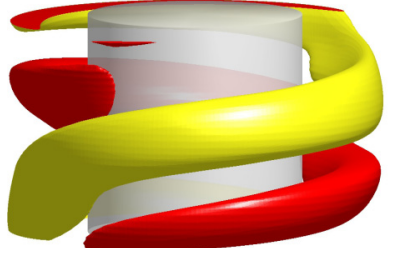

(4b)

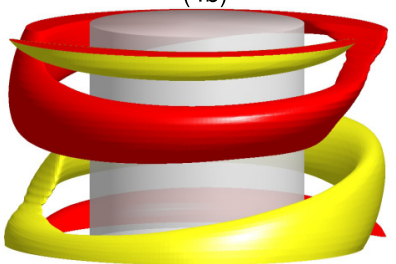

(1c) $[u(r, z), w(r, z)]$
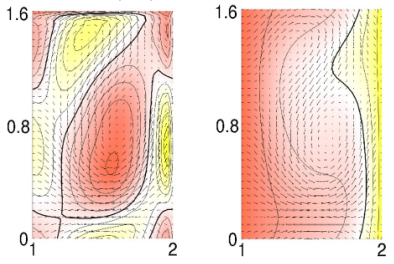

(2c)
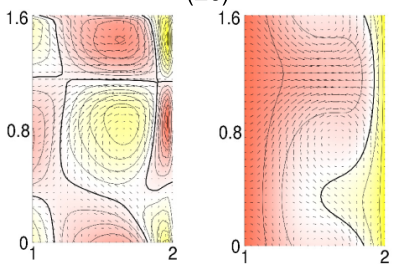

$(3 \mathrm{c})$
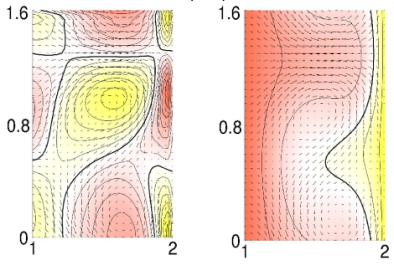

(4c)

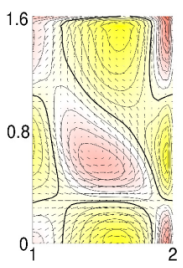

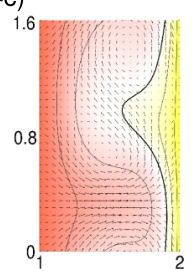

FIG. 13. Flow visualization of various flow pattern appearing within $1-\mathrm{mRIB}_{A \leftrightarrow B}^{t}$. Snapshots of the timedependent flow for (1) $1-\mathrm{mRIB}_{A}^{t}$, the transitional flows, (2) $\mathrm{M1}^{t}$-flow and (3) M(-1) ${ }^{t}$-flow, and (4) $1-\mathrm{mRIB}_{B}^{t}$. See Fig. 5 for further description. Control parameters: $\alpha=12, \operatorname{Re}_{i}=131$.

[60]. This is also supported by the randomness in persisting time and transitional flow between the two mixed ribbon states.

Although $E_{\text {kin }}$ does not go down to nearly zero, there does seem to be a significant drop within the transition process. In Fig. 6(a), the drop is from 2000 to 0 and in Fig. 10(a) from 4000 to 1000. The time-averaged spectrum for $1-\mathrm{mRIB}_{A \leftrightarrow B}^{t}[\mathrm{Fig} .10(\mathrm{c})]$ is qualitatively similar for $1-\mathrm{mRIB}^{t}[\mathrm{Fig}$. 8(c)], while the corresponding spectrum for $\mathrm{M} 1^{t}$-flow an $\mathrm{M}(-1)^{t}$-flow [Figs. 10(e) and 10(g)] clearly show the dominant transition modes $m=1$ and $m=-1$, respectively. Although it is more complex, the phase portrait of 1-mRIB ${ }_{A \leftrightarrow B}^{t}$ [Figs. 10(d), 10(f) and 10(h)] shows characteristics observed for 1-RIB R $_{\leftrightarrow}$ [Figs. 6(e)-6(h)]. Essentially one can identify two perpendicular orientated structures for $1-\mathrm{mRIB}_{A}^{t}$ and $1-\mathrm{mRIB}_{B}^{t}$. The observation of the 2-torus $1-\mathrm{mRIB}^{t}$ and the simpler heteroclinic cycle 1-RIB ${ }_{A \leftrightarrow B}^{t}$ suggest $1-\mathrm{mRIB}_{A}^{t}$ and $1-\mathrm{mRIB}_{B}^{t}$ are also tori.

The long time series of $\left|u_{m, n}\right|$ and $E_{\text {kin }}$ illustrated in Figure 11 support an intermittency scenario for $1-\mathrm{mRIB}_{A \leftrightarrow B}^{t}$. The persistence time $\tau_{p}$ within one of the solutions is random. However, a maximum persistence time $\tau_{p \text {, max }}$ can be observed, which seems to depend on the system parameter, 
in particular, the onset of the heteroclinic cycle 1-mRIB ${ }_{A \leftrightarrow B}^{t}$. With increasing distance to the onset the maximum persistence time $\tau_{p \text {, max }}$ decreases (Fig. 12), similar to the scenario for $1-\mathrm{RIB}_{A \leftrightarrow B}$. The schematic in Fig. 11(b) summarizes the transition behavior between $1-\mathrm{mRIB}_{A}^{t}$ and $1-\mathrm{mRIB}_{B}^{t}$ via the two potential transitional states $\mathrm{M} 1^{t}$-flow or $\mathrm{M}(-1)^{t}$-flow.

To look closer into the intermittency scenario, Fig. 12 presents the variation with $\operatorname{Re}_{i}$ of $\tau_{p \text {,max }}$ for 1-mRIB ${ }_{A \leftrightarrow B}^{t}$ in the parameter region presented in Fig. 2. The insets illustrate the scaling of $\tau_{p, \max }$ with control parameter $\epsilon$. For $\epsilon=\left|\operatorname{Re}_{i}-\operatorname{Re}_{i, c}^{A}\right|$, where $\operatorname{Re}_{i, c}^{A}$ is the critical values at which intermittency appears with increasing $\operatorname{Re}_{i}$ (from left in Fig. 12) and $\epsilon=\left|\operatorname{Re}_{i, c}^{B}-\operatorname{Re}_{i}\right|$, where $\operatorname{Re}_{i, c}^{B}$ is the critical values at which intermittency appears with decreasing $\operatorname{Re}_{i}$ (from right in Fig. 12). Both approaches suggest a type III intermittency $[61,62]$ based on the computation (circles) which can be approximated by $\propto \epsilon^{-1}$.

However, it should be noted that further analysis of the intermittency scenario is necessary. First the number of points for $\tau_{p, \max }$ for $1-\mathrm{mRIB}_{A \leftrightarrow B}^{t}$ are quite limited due to the very time intensive computations required. Second for the parameters studied, $1-\mathrm{mRIB}_{A \leftrightarrow B}^{t}$ is only present in a relatively narrow parameter island (Fig. 4). This makes a study of intermittency quite difficult. Changing a control parameter and departing from the onset on one side means approaching the onset from the other side. For further understanding future studies, in which 1-mRIB ${ }_{A \leftrightarrow B}^{t}$ is not limited to an island based on other system parameters, are required. A promising parameter to break up the parameter island of 1-mRIB ${ }_{A \leftrightarrow B}^{t}$ is the variation of the outer Reynolds number $\operatorname{Re}_{o}$.

Further visualization of the distinctive flow structures $1-\mathrm{mRIB}_{A}^{t}, 1-\mathrm{mRIB}_{B}^{t}$, M1 $1^{t}$-flow and $\mathrm{M}(-1)^{t}$ flow are presented in Fig. 13. The radial velocity $u(\theta, z)$ highlights the dominant contributions. The toroidally closed structure with dominant $m=0$ mode can be observed for $1-\mathrm{mRIB}_{A}^{t}$ and $1-\mathrm{mRIB}_{B}^{t}$ [Figs. 13(1,4)] as well as the helical left-winding shape for $\mathrm{M}^{t}{ }^{t}$-flow [Fig. 13(2)] and helical rightwinding shape for $\mathrm{M}(-1)^{t}$-flow [Fig. 13(3)].

\section{Stationary 1-mRIB}

Aside from the time-dependent 1-mRIB ${ }^{t}$ discussed previously (Figs. 3 and 4) we also detected simpler stationary 1-mRIBs. While RIBs are saddles and stationary in a comoving frame of reference, 1-mRIB are traveling waves. Figures 14 and 15 illustrate two examples of a stationary 1-mRIBs with dominant modulation modes either $m= \pm 2$ or $m= \pm 3$.

a. Stationary $1-\mathrm{mRIB}_{2}$. The stationary flow $1-\mathrm{mRIB}_{2}$ (Fig. 14) appears with increasing $\alpha$ at about $\alpha \approx 12.4$ when $1-\mathrm{mRIB}_{A \leftrightarrow B}^{t}$ disappears (cf. Fig. 3). The Fourier spectrum $(m, n)$ [Fig. 14(d)] shows the modes $m= \pm 2$ to be the second largest after the dominant ones, $m= \pm 1$. The corresponding flow structure is basically toroidal orientated with only minor modifications in the helical direction. That is, modifications in the contours of $u(r, z)$ are significantly smaller than for 1-mRIB ${ }^{t}$ [Fig. 9(1,2)].

b. Stationary $1-\mathrm{mRIB}_{3}$. With increasing $\alpha, 1-\mathrm{mRIB}_{3}$ evolves out of $1-\mathrm{mRIB}_{2}^{s}$ at $\alpha \approx 15.2$ (Fig. 3) when the modes $m= \pm 3$ become finite and become the second largest modes [Fig. 15(d)]. These dominant $m= \pm 3$ modes result in stronger modulation of the flow structure, e.g. they are visible in $u(r, z)$ and in the isosurfaces of $\eta$ [Figs. 15(a) and 15(b)].

Similarly to their more complex time-dependent cousins $1-\mathrm{mRIB}_{t}, 1-\mathrm{mRIB}$ can also alternate between the two states $1-\mathrm{mRIB}_{A}$ and $1-\mathrm{mRIB}_{B}$ (Fig. 3). The transition scenario for $1-\mathrm{mRIB}_{A \leftrightarrow B}$ is similar to that for $1-\mathrm{RIB}_{A \leftrightarrow B}$ (Fig. 6). The transitional flow is mainly a modulated M0-flow, but with additional mode contributions $(m \neq 0)$. Despite the additional modes $|m|>1$ we never observed the helical flow structure as discovered for the time-dependent scenario $1-\mathrm{mRIB}_{A \leftrightarrow B}^{t}$. The heteroclinic connection is also nonoscillatory in this case [60]. As described previously this modulated M0-flow (here including $m \neq 0$ ) is different to the classical closed TVF structure with $m=0$. As in the previous scenario for alternating $1-\mathrm{RIB}_{A \leftrightarrow B}$ no toroidally closed vortex structure can be observed. Moreover, as for $1-\mathrm{RIB}_{A \leftrightarrow B}$ the persistence time for $1-\mathrm{mRIB}_{A \leftrightarrow B}$ is determined by system parameters and decreases with distance to the onset. 
(a) $u(r, \theta)$

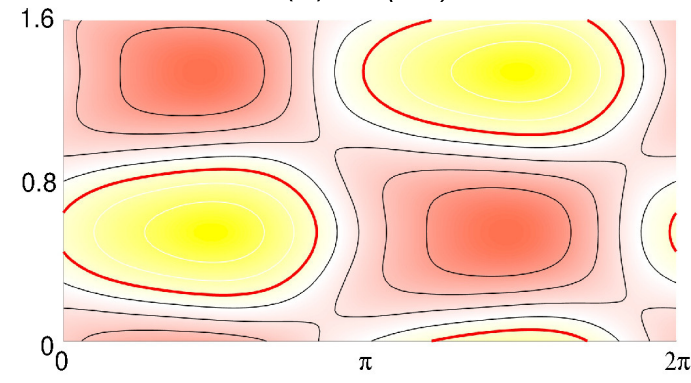

(c) $[u(r, z), w(r, z)]$

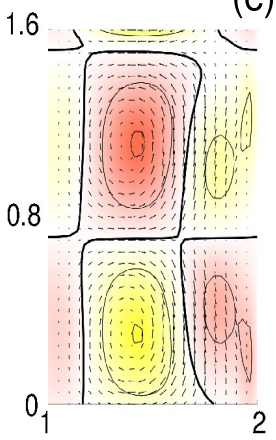

(b) $\eta=+140$

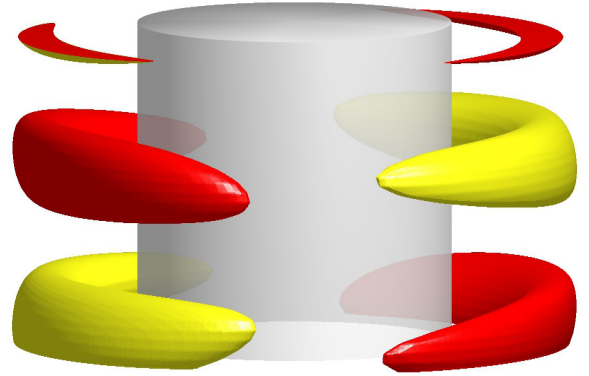

(d)

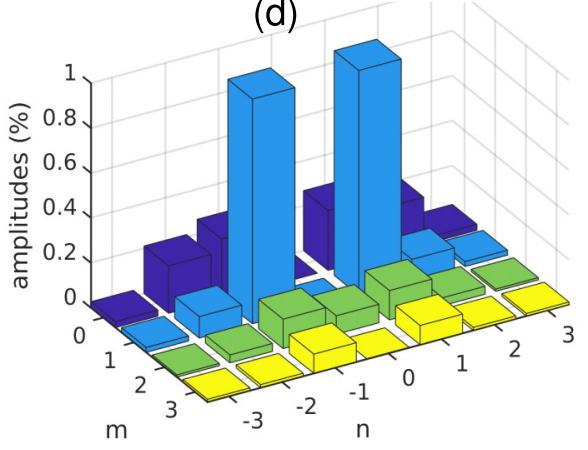

FIG. 14. Stationary 1-mRIB 2 . Flow visualization and specification of $1-\mathrm{mRIB}_{2}$. Shown are (a) the radial velocity $u(\theta, z)$ on an unrolled cylindrical surface in the annulus at mid-gap [red (yellow) color indicates in (out) flow], (b) isosurfaces of $\eta$ [red (dark gray) and yellow (light gray) colors correspond to positive and negative values, respectively, with zero specified as white], and (c) vector plot $[u(r, z), w(r, z)]$ of the radial and axial velocity components [including color-coded azimuthal vorticity $\eta$ (left) and azimuthal velocity $v$ (right)]. The thick contour lines on the left and right correspond to $\eta=0$ (left) and $v / \operatorname{Re}_{i}=0.5$ (right), respectively. (d) Fourier spectrum $(m, n)$. Control parameters: $\alpha=15, \operatorname{Re}_{i}=124$.

\section{Angular momentum transport}

To further characterize the effect of radial mass flux, we examine the behavior of the angular momentum and torque for a variety of flow states and different $\alpha$. Figure 16(a) shows the mean (axially and azimuthally averaged) angular momentum $L(r)=r\langle v(r)\rangle_{\theta, z} / \operatorname{Re}_{i}$ as a function of radius $r$ for structures and $\alpha$ as indicated in the caption. Except for $\alpha=0$ all curves show a similar shape. In general, the profiles indicate typical behavior in that $L(r)$ decreases monotonically and is positive near the inner cylinder and negative near the outer one, with a slowly varying section in between. With increasing $\alpha$ the profiles of $L(r)$ become more strongly curved, while the zero-crossing $\left[L\left(r_{0}\right)=0\right.$ ] is displaced outwards [inset in Fig. 16(a): $r_{0}$ with $\alpha$ ]. Moreover, the plateaulike region in the central region of the bulk becomes more pronounced as it moves to higher values. Simultaneously with increasing $\alpha$ the variation of the profiles starting at the inner cylinder decreases, the slope becomes more shallow. The opposite holds for the variation of profiles on the outer cylinder; with increasing $\alpha$ the slope increases. In addition, the slopes at the point of zero-crossing $\delta\left(r_{0}\right)=\partial\left[L\left(r_{0}\right)\right] / \partial r$ [inset in Fig. 16(a)] essentially decrease almost linearly with $\alpha$. At the same time the induced rotation at the inner cylinder is preserved for larger distance into and through the bulk width. Interestingly the profiles for TVFs and SPIs at the same value $\alpha$ are very similar with slightly more visible belly shape characteristics in case of helical SPIs.

Figure 16(b) illustrates the dimensionless torque $G(r)=v J^{\omega}$ within the annulus. In calculating the torque we used the fact that for a flow between infinite cylinders the transverse current of the 
(a) $u(r, \theta)$

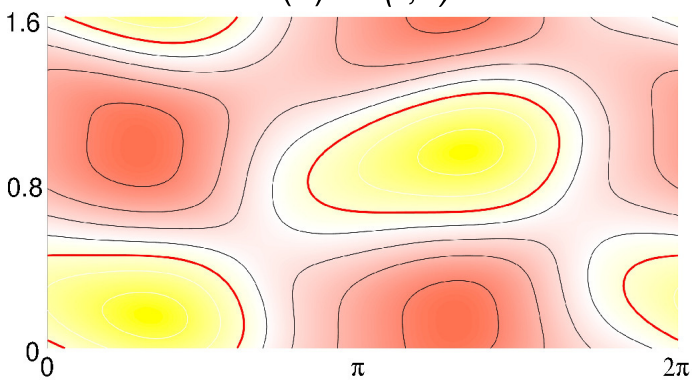

(c) $[u(r, z), w(r, z)]$

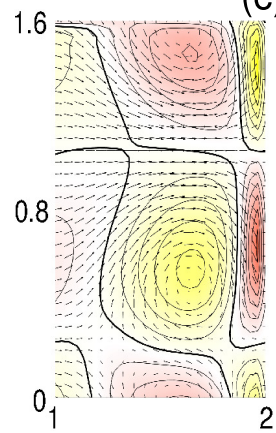

(b) $\eta=+140$

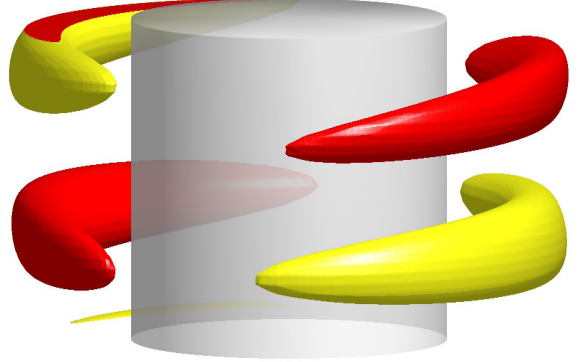

(d)

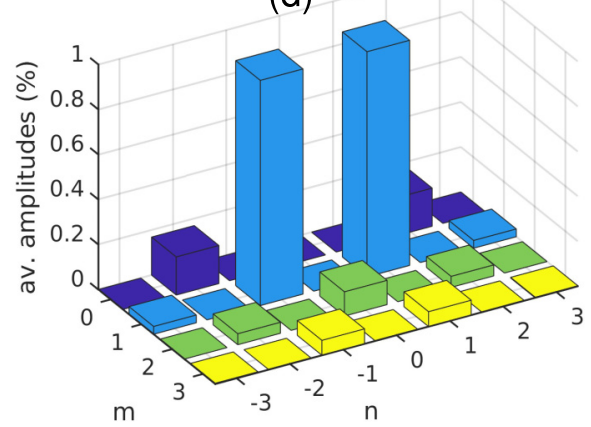

FIG. 15. Stationary 1-mRIB 3 . Flow visualization and specification of 1-RIB 3 . Like Fig. 14, but for control parameters $\alpha=16, \operatorname{Re}_{i}=124$.

azimuthal motion $J^{\omega}=r^{3}\left[\langle u \omega\rangle_{A, t}-v\left\langle\partial_{r} \omega\right\rangle_{A, t}\right]$ (with $\langle\ldots\rangle_{A} \equiv \int \frac{r d \theta d z}{2 \pi r l}$ ), is a conserved quantity [63]. Like the angular momentum, the profiles of $G(r)$ change as $\alpha$ is increased from an almost flat profile for $\alpha=0$ toward an S-curve shape for larger $\alpha$ with significant increase in slope and absolute value toward the outer cylinder. The result of this is also evident in the total torque $G_{\text {total }}$, which increases almost linearly with $\alpha$ [inset in Fig. 16(b)].
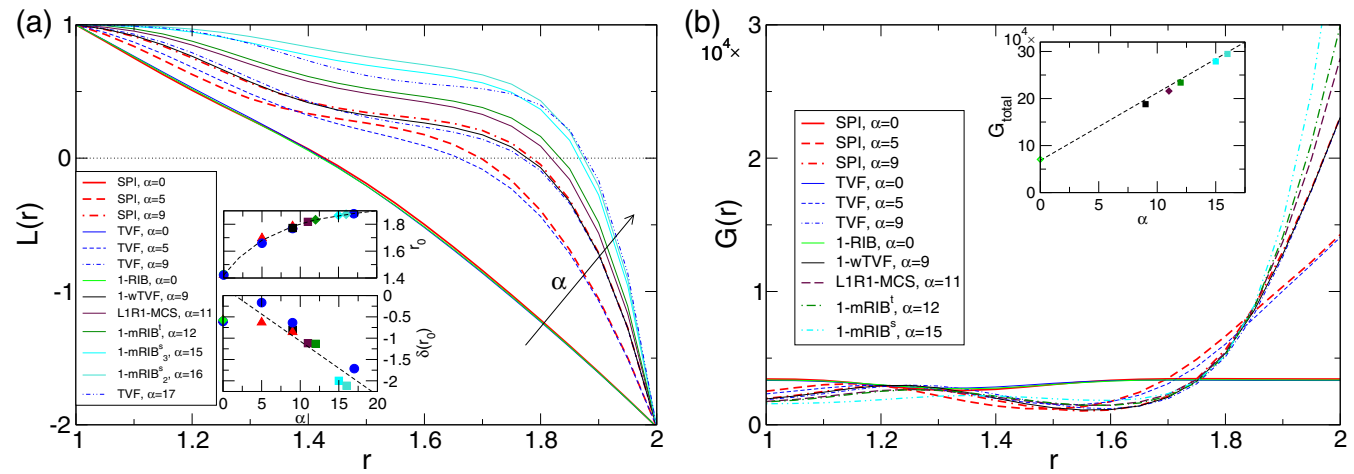

FIG. 16. Variation in (a) mean (axially and azimuthally averaged) angular momentum $L(r)=$ $r\langle v(r)\rangle_{\theta, z} / \operatorname{Re}_{i}$ and (b) dimensionless torque $G=v J^{\omega}$ (see text for details) versus the radius $r$ for solutions with increasing $\alpha$ as indicated at $\mathrm{Re}_{i}=124$. The insets in (a) show the variation with $\alpha$ for root $r_{0}$ of $L$ and corresponding slopes $\delta=\partial\left[L\left(r_{0}\right)\right] / \partial r$. The inset in (b) illustrates the dependence of the total torque $G_{\text {total }}$ on $\alpha$. 


\section{DISCUSSION AND CONCLUSION}

For more than a century the Taylor-Couette system has been extensively used in both computational and experimental studies and has proven itself as a foundational paradigm of fluid dynamics. Over the years various modifications of the basic system have been formulated to investigate specific aspects. One of them is to consider radial mass flux through porous cylinder walls. In this paper we have investigated the influence of radial muss flux (measured by the radial Reynolds number $\alpha$ ) in wide gap Taylor-Couette flow with counter-rotating cylinders. This resulted in a more detailed picture of the effect of a radial flow and its interaction with helical solutions.

The main results can be summarized as follows.

(1) Detection of stable ribbons (1-RIBs) at low Reynolds numbers with the smallest (helical) azimuthal wave number $m= \pm 1$ [4]. To date the only stable RIBs reported have higher azimuthal wave number $m= \pm 2$ (2-RIB) [46,47].

(2) Detection of stable mixed ribbons (1-mRIBs) as well as stable mixed-cross-spirals (MCS) [48] without symmetry restrictions (e.g., subspaces [48,49]). Thereby mRIBs can appear either stationary $(1-\mathrm{mRIB})$ or time-dependent $\left(1-\mathrm{mRIB}^{t}\right)$.

(3) Depending on various system parameters unstable RIB and mRIB are found. We detected heteroclinic cycles between two saddles $1-\mathrm{RIB}_{A \leftrightarrow B}, 1-\mathrm{mRIB}_{A \leftrightarrow B}$ as well as alternation 1-mRIB $\mathrm{m}_{A \leftrightarrow B}^{(t)}$.

(4) The heteroclinic connection between the two symmetrically related states can be either of oscillatory type (for 1-mRIB ${ }_{A \leftrightarrow B}^{(t)}$ ) or of nonoscillatory type (for 1-R $\mathrm{RB}_{A \leftrightarrow B}$ ) [60].

(5) For 1-mRIB ${ }_{A \leftrightarrow B}^{t}$ a more complex intermittency scenario is observed, which seems to be type III intermittency [62].

(6) The $\left(\mathrm{Re}_{i}, \alpha\right)$ parameter space (Fig. 4) illustrates a rich variety of flow structures.

Depending on the type of heteroclinic connection, oscillatory or nonoscillatory, the transitional flow is different. For stationary solutions, $1-\mathrm{RIB}_{A \leftrightarrow B}$ and $1-\mathrm{mRIB}_{A \leftrightarrow B}$, the connection between the saddles is of nonoscillatory type and the flow evolves via a M0-flow with azimuthal mode $m=0$. We found the persistence time within and transition time between the two symmetrically related 1-(m)RIBs to be almost constant depending only on control parameters (e.g., $\alpha$ and $\mathrm{Re}_{i}$ ). The transitional M0-flow [see Fig. 5(2)]is unrelated to the classical TVF, which is also $m=0$, but consists of toroidal vortices. No structures similar to toroidal vortices appear in the M0-flow.

Contrary to the alternation for $1-\mathrm{mRIB}_{A \leftrightarrow B}$, for $1-\mathrm{mRIB}_{A \leftrightarrow B}^{t}$ the transition includes various oscillating dynamics as here the two saddles are linked via a heteroclinic connection of oscillatory type. As a result the transition itself appears via time-dependent helical flow states, with either leftor right-winding dominance, $\mathrm{M} 1^{t}$-flow and $\mathrm{M}(-1)^{t}$-flow, respectively. Contrary to the situation of the stationary alternation, the heteroclinic cycles $1-\mathrm{RIB}_{A \leftrightarrow B}$ and $1-\mathrm{mRIB}_{A \leftrightarrow B}$ do not display a simple correlation between control parameters and transition or persistence time. The transition between these solutions related by symmetry appears randomly. However, a maximum persistence time predetermined by the system parameters can be identified for the heteroclinic cycle $1-\mathrm{mRIB}_{A \leftrightarrow B}^{t}$. The simulations suggest an intermittency scenario of type III, but further investigations are necessary to fully understand this behavior. Similarly the transitional flow is also random, occurring via either $\mathrm{M} 1^{t}$-flow or M(-1) ${ }^{t}$-flow [Fig. 11(a)]. 1-RIB and 1-mRIB were identified as limit cycles whereas time-dependent 1 -mRIB ${ }^{t}$ live on a two-torus invariant manifold.

Further interesting questions emerging from the present work are the effect of the boundary conditions on the stability and flow characteristics. Gallaire and Chomaz [64] showed that the stability may depend on the boundary conditions imposed at the inlet and the outlet. A concrete question that arises in this context is that of a nonpermeable nonpermeable outer cylinder. In such a setup an axial flow is needed to evacuate the additional mass entering radially. This is of particular interest, as continuous working filtration devices have this configuration.

We hope that our computational results will encourage further experimental works with the aim of controlling flows with further potential for application, e.g., flow separation devices for industrial filtration applications [21-25], e.g., oil-sand separation [13] or extraction of blood plasma [15]. The present work has not only revealed the onset of different instabilities, but moreover has studied 
solutions, of which the vortex structure is azimuthally dominated or helically dominated, together with their topology within the parameter space. The spatio-temporal behavior of these flows is of particular interest and could be used to specialize and personalize different applications with respect to filtration and separation.

However, our results on helical flow structures, and their interaction to form (mixed) ribbons under radial flow conditions may be relevant to astrophysical flows. It may shed some light on experimentally observed physical phenomena. Examples are the formation of strong rotating flows produced by a rotating disk [33] and polar vortex flow in the Earth's atmosphere [32,34].

\section{ACKNOWLEDGMENTS}

S.A. acknowledges support as Serra Húnter Fellow. We are grateful to the anonymous referees whose comments resulted in substantial improvement of the original manuscript.

\section{APPENDIX}

This section provides a description of the correlation between the azimuthal wave number $M$ (see Table I) and the 2D Fourier spectrum in Eqs. (2.4) and (2.5). To motivate this we will focus on SPI (RIB are analogous).

In a comoving frame, the SPI is effectively one dimensional and stationary. The corresponding fields do not separately depend on coordinates $\theta, z$ and time $t$, instead they are linked via a combined phase variable,

$$
\Theta=k z+M \theta-\omega(k, M) t
$$

( $k$ and $M$ are the axial and azimuthal wave numbers, respectively, and $\omega$ is the frequency).

Thus, using the combined phase variable $f(r, \theta, z, t)=F(r, \Theta)$ [cf. Eq. (A1)] and comparing the Fourier decompositions

$$
f(r, \theta, z, t)=\sum_{m, n} f_{m, n}(r, t) e^{i(m \theta+n k z)}=\sum_{\nu} F_{\nu}(r) e^{i \nu \Theta},
$$

one finds

$$
f_{m, n}(r, t)=\delta_{m, n M} e^{-i n \omega t} F_{n}(r) .
$$

As a result only the mode combinations $m=n M$ appear in the 2D Fourier spectrum of a SPI with azimuthal wave number $M$.

Figure 17 illustrates the $\theta-z$ plane of an unrolled cylindrical surface. Here lines of constant phase, $\Theta=\Theta_{0},\left(r=\right.$ const. $\left.z_{0}=-\frac{M}{k} \theta+\frac{\omega(k, M)}{k} t+\frac{1}{k} \Theta_{0}\right)$ are straight with constant slope $-M / k$. With the convention of taking $k$ to be positive an azimuthal wave number $M>0$ implies a left handed spiral [L-SPI, Fig. 17(a)] while $M<0$ refers to a right handed spiral [R-SPI, Fig. 17(c)]. Under the operation $z \rightarrow-z$, both L-SPI and R-SPI are mirror images of each other. Thus, the two symmetrically related solutions L1-SPI $(M=1)$ and R1-SPI $(M=-1)$ can be identified by $\left|u_{1,1}\right|$ $\left(\left|u_{-1,-1}\right|\right)$ and $\left|u_{-1,1}\right|\left(\left|u_{1,-1}\right|\right)$ [cf. Eq. (A3)].

Lines of constant phase and with them the whole spiral structure rotates in the azimuthal direction $\theta$ with an angular velocity $\dot{\theta}=\frac{\omega}{M}$. Further using Eq. (A1) the axial phase velocity is given by $w_{p h}=$ $\frac{\omega}{k}=\frac{M}{k}=\dot{\theta}$, which for L-SPI $(M>0)$ is positive and for R-SPI $(M<1)$ is negative. For 1-RIB as a linear superposition of L1-SPI and R1-SPI the lines of constant phase in the $\theta-z$ plane [Fig. 17(b)]are horizontal characterizing 1-RIB as standing wave in the axial direction, while rotating azimuthally.

The bottom of Fig. 17 shows the two-dimensional Fourier mode space spanned by the azimuthal and axial Fourier mode indices $m$ and $n$. Blue squares with red or orange circles denote linearly driven modes and, respectively, superimpose to linear Fourier mode subspaces indicated by thick lines. They represent linear subspace for L1-SPI $(m=n, n)$ in Fig. 17(a) and the linear subspace 
(a) $L 1-S P \mid$
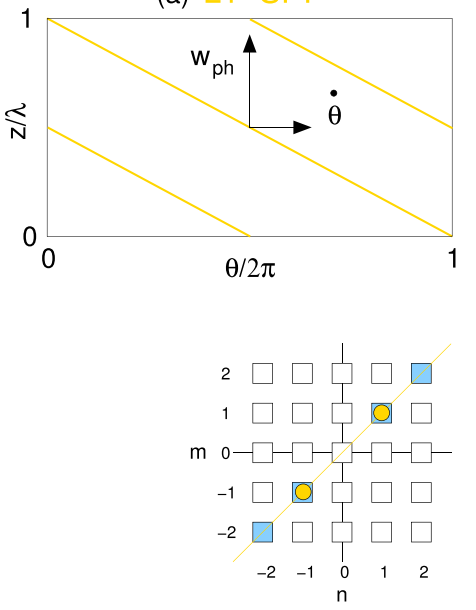

(b) $1-\mathrm{RIB}$

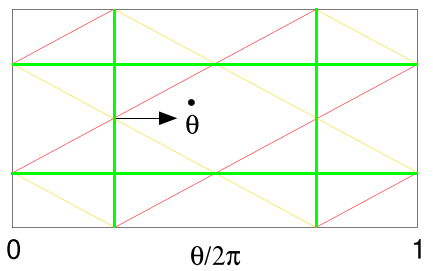

(c) R1-SPI

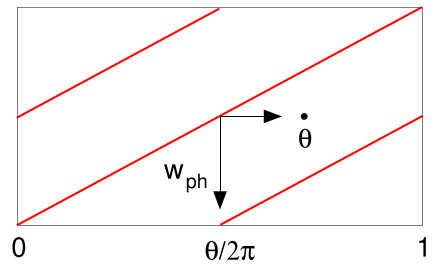

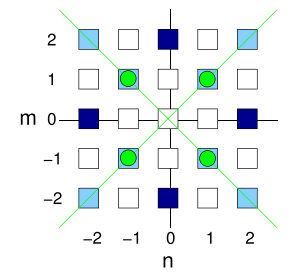

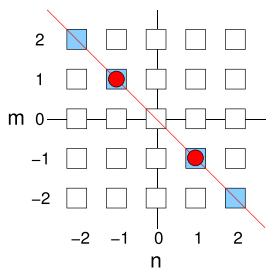

FIG. 17. Characterization of various structures. (a) L1-SPI (orange), (b) 1-RIB (green), and (c) R1-SPI (red). Top: lines of constant phase $\Theta=0$ on an azimuthally unrolled cylindrical surface ( $\theta-z$ plane) at mid-gap. Arrows indicate their velocities. Bottom: two-dimensional Fourier mode space spanned by the azimuthal and axial Fourier mode indices $m$ and $n$. Blue squares with red or orange circles denote linearly driven modes and lead to to linear Fourier mode subspaces indicated by thick lines. They represent linear L1-SPI $(m=n, n)$ in panel (a) and linear R1-SPI $(m=-n, n)$ in panel (c). Blue squares in panel (b) without a circle indicate nonlinearly driven modes in 1-RIB.

for R1-SPI $(m=-n, n)$ in Fig. 17(c). Dark blue squares in Fig. 17(b) without a circle indicate nonlinearly driven modes in 1 -RIB. Note, that for 1 -RIB the modes $(0, \pm 1)$ are zero. In difference with 1 -mRIB the modes $(0, \pm 1)$ are finite.

[1] G. I. Taylor, Stability of a viscous liquid contained between two rotating cylinders, Philos. Trans. R. Soc. London A 223, 289 (1923).

[2] S. Chandrasekhar, Hydrodynamic and Hydromagnetic Stability (Clarendon Press, Oxford, 1961).

[3] R. C. DiPrima and H. L. Swinney, Instabilities and transition in flow between concentric rotating cylinders, in Hydrodynamic Instabilities and the Transition to Turbulence, edited by H. L. Swinney and J. G. Gollub, Vol. 45 in Topics in Applied Physics (Springer, Berlin, 1985).

[4] R. Tagg, The Couette-Taylor problem, Nonlin. Sci. Today 4, 1 (1994).

[5] V. I. Belinicher and V. S. L'vov, A scale-invariant theory of fully developed hydrodynamic turbulence, Zh. Eksp. Teor. Fiz. 93, 533 (1987).

[6] R. H. Kraichnan, Models of Intermittency in Hydrodynamic Turbulence, Phys. Rev. Lett. 65, 575 (1990).

[7] C. Beck, Superstatistics in hydrodynamic turbulence, Physica D: Nonlin. Phenom. 193, 195 (2004).

[8] Y. Li and C. Meneveau, Origin of Non-Gaussian Statistics in Hydrodynamic Turbulence, Phys. Rev. Lett. 95, 164502 (2005).

[9] G. L. Eyink and K. R. Sreenivasan, Onsager and the theory of hydrodynamic turbulence, Rev. Mod. Phys. 78, 87 (2006).

[10] G. Beadoin and M. Y. Jaffrin, Plasma filtration in Couette flow membrane devices, Artif. Organs 13, 43 (1989).

[11] A. Schwille, D. Mitra, and M. Lueptow, Design parameters for rotating cylindrical filtration, J. Membr. Sci. 204, 53 (2002). 
[12] S. Lee and R. M. Lueptow, Rotating membrane filtration and rotating reverse osmosis, J. Chem. Eng. Jpn. 37, 471 (2004).

[13] J. Masliyah, Z. J. Zhou, Z. Xu, J. Czarnecki, and H. Hamza, Understanding water-based bitumen extraction from Athabasca oil sands, Can. J. Chem. Eng. 82, 628 (2004).

[14] C. D. Andereck, S. S. Liu, and H. L. Swinney, Flow regimes in a circular Couette system with independently rotating cylinders, J. Fluid Mech. 164, 155 (1986).

[15] R. M. Lueptow and A. Hajiloo, Flow in a rotating membrane plasma separator, Trans. Am. Soc. Artif. Intern. Organs 41, 182 (1995).

[16] K. Ohashi, K. Tashiro, F. Kushiya, T. Matsumoto, S. Yoshida, M. Endo, T. Horio, K. Ozawa, and K. Sakai, Rotation-induced Taylor vortex enhances filtrate flux in plasma separation, Trans. Am. Soc. Artif. Intern. Organs 34, 300 (1988).

[17] K. H. Kroner, V. Nissinen, and H. Ziegler, Improved dynamic filtration of microbial suspensions, Bio/Technology 5, 921 (1987).

[18] K. H. Kroner and V. Nissinen, Dynamic filtration of microbial suspensions using an axially rotating filter, J. Membr. Sci. 36, 85 (1988).

[19] B. Hallström and M. Lopez-Leiva, Description of a rotating ultrafiltration module, Desalination 24, 273 (1978).

[20] W. Tobler, Dynamic filtration-the engineering concept of the Escher Wyss pressure filter, Filtr. Sep. 15, 630 (1979).

[21] H. B. Winzeler and G. Belfort, Enhanced performance for pressure-driven membrane processes: The argument for fluid instabilities, Membr. Sci. 80, 35 (1993).

[22] A. Margaritis and C. R. Wilke, The rotorfermentor. I. Description of the apparatus, power requirements, and mass transfer characteristics, Biotechnol. Bioeng. 20, 709 (1978).

[23] W. Tobler, Dynamic filtration: Principle and application of shear filtration in an annular gap, Filtr. Sep. 19, 329 (1982).

[24] T. Murase, E. Iritani, P. Chidphong, K. Kano, K. Atsumi, and M. Shirato, High speed microfiltration using a rotating, cylindrical ceramic membrane, Int. Chem. Eng. 31, 370 (1991).

[25] S. Wroński, E. Molga, and L. Rudniak, Dynamic filtration in biotechnology, Bioprocess Eng. 4, 99 (1989).

[26] K. Min and R. M. Lueptow, Hydrodynamic stability of viscous flow between rotating porous cylinders with radial flow, Phys. Fluids 6, 144 (1994).

[27] E. Serre, M. A. Sprague, and R. M. Lueptow, Stability of Taylor-Couette flow in a finite-length cavity with radial through-flow, Phys. Fluids 20, 034106 (2008).

[28] D. Martinand, E. Serre, and R. Lueptow, Absolute and convective instability of cylindrical Couette flow with axial and radial flows, Phys. Fluids 21, 104102 (2009).

[29] D. Martinand, E. Serre, and R. Lueptow, Linear and weakly nonlinear analyses of cylindrical Couette flow with axial and radial flows, JFM 824, 438 (2017).

[30] E. C. Johnson and R. M. Lueptow, Hydrodynamic stability of flow between rotating porous cylinders with radial and axial flow, Phys. Fluids 9, 3687 (1997).

[31] B. Gallet, C. R. Doering, and E. A. Spiegel, Destabilizing Taylor-Couette flow with suction, Phys. Fluids 22, 034105 (2010).

[32] E. Kersale, D. W. Hughes, G. I. Ogilvie, S. M. Tobias, and N. O. Weiss, Global magnetorotational instability with inflow. I. Linear theory and the role of boundary conditions, Astrophys. J. 602, 892 (2004).

[33] R. R. Kerswell, Instability driven by boundary inflow across shear: A way to circumvent Rayleighs stability criterion in accretion disks? J. Fluid Mech. 784, 619 (2015).

[34] M. E. McIntyre, The stratospheric polar vortex and sub-vortex: Fluid dynamics and midlatitude ozone loss, Philos. Trans. R. Soc. London, Ser. A 352, 227 (1995).

[35] S. A. Balbus and J. F. Hawley, Instability, turbulence, and enhanced transport in accretion disks, Rev. Mod. Phys. 70, 1 (1998).

[36] S. K. Bahl, Stability of viscous flow between two concentric rotating porous cylinders, Def. Sci. J. 20, 89 (2014).

[37] S. Chang and W. K. Sartory, Hydromagnetic stability of dissipative flow between rotating permeable cylinders, J. Fluid Mech. 27, 65 (1967). 
[38] A. A. Kolyshkin and R. Vaillancourt, Convective instability boundary of Couette flow between rotating porous cylinders with axial and radial flows, Phys. Fluids 9, 910 (1997).

[39] K. Ilin and A. Morgulis, On the stability of the Couette-Taylor flow between rotating porous cylinders with radial flow, European J. Mech. - B/Fluids 80, 174 (2020).

[40] C. A. Jones, The transition to wavy Taylor vortices, J. Fluid Mech. 157, 135 (1985).

[41] C. Hoffmann, S. Altmeyer, A. Pinter, and M. Lücke, Transitions between Taylor vortices and spirals via wavy Taylor vortices and wavy spirals, New J. Phys. 11, 053002 (2009).

[42] M. Golubitsky and W. F. Langford, Pattern formation and bistability in flow between counter-rotating cylinders, Physica D 32, 362 (1988).

[43] C. Hoffmann and M. Lücke, Spiral Vortices and Taylor Vortices in the Annulus between Counter-rotating Cylinders (Springer Verlag, Berlin, 2000), p. 55.

[44] P. Chossat and G. Iooss, The Couette-Taylor Problem (Springer, Berlin, 1994).

[45] R. Tagg, W. S. Edwards, H. L. Swinney, and P. S. Marcus, Nonlinear standing waves in Couette-Taylor flow, Phys. Rev. A 39, 3734(R) (1989).

[46] A. Pinter, M. Lücke, and Ch. Hoffmann, Bifurcation of standing waves into a pair of oppositely traveling waves with oscillating amplitudes caused by a three-mode interaction, Phys. Rev. E 78, 015304(R) (2008).

[47] A. Pinter, M. Lücke, and Ch. Hoffmann, Wave-number dependence of the transitions between traveling and standing vortex waves and their mixed states in the Taylor-Couette system, Phys. Rev. E 78, 017303 (2008).

[48] S. Altmeyer and C. Hoffmann, Secondary bifurcation of mixed-cross-spirals connecting travelling wave solutions, New J. Phys. 12, 113035 (2010).

[49] S. Altmeyer and C. Hoffmann, On secondary instabilities generating footbridges between spiral vortex flow, Fluid Dyn. Res. 46, 025503 (2014).

[50] M. Krupa, Robust heteroclinic cycles, J. Nonlin. Sci. 7, 129 (1997).

[51] M. Krupa and I. Melbourne, Asymptotic stability of heteroclinic cycles in systems with symmetry, Ergod. Th. Dyn. Sys. 15, 121 (1995).

[52] A. S. Kuznetsov and J. Kurths, Stable heteroclinic cycles for ensembles of chaotic oscillators, Phys. Rev. E 66, 026201 (2002).

[53] D. Armbruster, J. Guckenheimer, and P. Holmes, Heteroclinic cycles and modulated travelling waves in systems with O(2) symmetry, Physica D 29, 257 (1988).

[54] I. Melbourne, P. Chossat, and M. Golubitsky, Heteroclinic cycles involving periodic solutions in mode interactions with O(2) symmetry, Proc. R. Soc. Edinburgh A 113, 315 (1989).

[55] J. Guckenheimer and P. Holmes, Structurally stable heteroclinic cycles, Math. Proc. Cambridge Philos. Soc. 103, 189 (1988).

[56] S. Hughes and A. Randriamampianina, An improved projection scheme applied to pseudospectral methods for the incompressible NavierStokes equations, Int. J. Numer. Methods Fluids 28, 501 (1998).

[57] I. Mercader, O. Batiste, and A. Alonso, An efficient spectral code for incompressible flows in cylindrical geometries, Comput. Fluids 39, 215 (2010).

[58] S. Altmeyer, C. Hoffmann, M. Heise, J. Abshagen, A. Pinter, M. Lücke, and G. Pfister, End wall effects on the transitions between Taylor vortices and spiral vortices, Phys. Rev. E 81, 066313 (2010).

[59] S. Altmeyer, Non-linear dynamics and alternating "flip" solutions in ferrofluidic Taylor-Couette flow, J. Magn. Magn. Mater. 452, 427 (2018).

[60] Y. Bengana and L. Tuckerman, Spirals and ribbons: Frequencies from mean flows and heteroclinic orbits, Phys. Rev. Fluids 4, 044402 (2019).

[61] Y. Pomeau and P. Manneville, Intermittent transition to turbulence in dissipative dynamical systems, Commun. Math. Phys. 74, 189 (1980).

[62] W. M. Macek and M. Strumik, Hydromagnetic Intermittent Convection in a Magnetized Viscous Fluid, Phys. Rev. Lett. 112, 074502 (2014).

[63] B. Eckhardt, S. Grossmann, and D. Lohse, Torque scaling in turbulent Taylor-Couette flow between independently rotating cylinders, J. Fluid Mech. 581, 221 (2007).

[64] F. Gallaire and J. M. Chomaz, The role of boundary conditions in a simple model of incipient vortex breakdown, Phys. Fluids 16, 274 (2004). 